\title{
LES TEMPS DU TRAVAIL ET CEUX DE LA CONSOMMATION ALIMENTAIRE. SYSTĖMES DE PRODUCTION ÉCONOMIQUE ET DE POUVOIR DANS LES MONASTẼRES DU HAUT MOYEN ÂGE EN ITALIE DU CENTRE-NORD (VII ${ }^{\mathrm{e}}-\mathrm{XI}^{\mathrm{e}}$ SIÈCLES)
}

\section{PAOLO DE VINGO}

UDC: $27-788-055.1$ 331.103 .252

Original scientific paper

Manuscript received: 17. 01. 2017.

Revised manuscript accepted: 20. 02. 2017.

DOI: 10.1484/J.HAM.5.113730

\author{
P. de Vingo \\ Università di Torino \\ Dipartimento di Studi Storici \\ Sezione di Archeologia \\ via S. Ottavio 20 \\ 10124 Torino, Italia
}

The monks did not work the fields themselves; they only helped farm in cases of dire need. There was a tendency to entrust farm tasks to serfs-who were nearly slaves - and to farmers. As of the 10th century the latter were referred to as lay brothers, laymen who joined the monks. The lay brothers, their actions, and their need to work to earn their daily bread, to see to the community's subsistence, yielded consequences essential to the formation of postRoman European civilization. Serfs and lay brothers began tilling, irrigating, and draining the land around the monasteries to plant that necessary to their survival. They developed grain farming and zootechnics, cut down woods, and drained marshes, dug canals to irrigate the fields, planted vineyards, and prepared the ground for a type of economy that in the Early Middle Ages would classify as sustainable and perfectly integrated in an equal balance of resources and production capacity. Thus, it was the monks that sparked the development of an economic system, which provided adequate means and resources to organize fairs and markets in certain periods and to control important import-export activities. The profits were then used to purchase sculptures, paintings, gold objects, and precious stones.

Keywords: Material culture, monastic diet, pottery, economic production and organisation

\section{AVANT-PROPOS}

La première moitié du VI ${ }^{\mathrm{e}}$ siècle est considérée comme la période où le monachisme occidental s'est amplement propagé, après la phase d'abandon des formes de vie érémitique caractérisée par le modèle contenu dans la Vita Martini de Sulpice-Sévère ${ }^{1}$. Cependant, on peut affirmer que, tout au moins jusqu’à Grégoire Le Grand, deux formes de vie monastique cohabitèrent sur la péninsule italienne : la forme érémitique, désormais en déclin et la forme cénobitique, qui s’inspirait des Règles, composées par des personnages à la forte personnalité, en vue de faciliter la prière et la vie en commun des hommes de religion. À cette date, si l'on excepte les règles insulaires qui ne seront connues grâce à Colomban qu'à la fin du VIe siècle, les deux Règles principales répandues dans les aires européennes occidentales, étaient celles du "Maître" et de Benoît de Nursie, deux abbés qui élaborèrent une synthèse innovante de toutes les expériences passées².

Pour des renvois normatifs, on peut rapprocher ces deux Règles des Dialogues et de la Correspondance de Grégoire le Grand et percevoir ainsi la situation de ceux qui avaient adhéré aux principes de la vie monastique en Italie. S’il existait encore des ermites fixés sur les îles du centre-nord de la mer Tyrrhénienne et en Tuscie, on notait aussi la présence de moines non sédentaires, les "sarabaïtes", contre lesquels Benoît de Nursie lança de très graves accusations. Sa vision monastique était entièrement fondée sur la stabilitas des religieux vivant dans l'espace du coenobium, seul milieu où il était possible de conjuguer les deux finalités fondamentales de sa Règle - la prière continue et le travail, comme moment de sacrifice 3 . Grégoire le Grand fut si fasciné et convaincu par la cohérence, le contenu des principes bénédictins et l'équilibre de l'organisation sociale des cénobes, que, non content de leur consacrer tout le second livre de ses Dialogues, son appui à la Règle de saint Benoît lui donna de s'affirmer dans le monde monastique italien au cours des VII ${ }^{\mathrm{e}}-\mathrm{VIII}{ }^{\mathrm{e}}$ siècles ${ }^{4}$.

Rappelons que la Règle bénédictine, élaborée dans une période de forte crise politique et militaire, dans une péninsule italienne dévastée par les guerres gothiques (535-553), planifiait l'ensemble de la journée monastique. Obéissance, silence et humilité étaient les fondements de ces préceptes, requis et parfois imposés par des châtiments corporels voire par l'excommunication's. Les moines devaient se lever en pleine nuit pour prier, lire et chanter les Psaumes ; ils étaient ensuite appelés à tour de rôle à exécuter des tâches agricoles, culinaires ou de copie au scriptorium ${ }^{6}$.

La sobriété et le respect des mesures alimentaires sont au centre de nombreux débats et controverses. La consistance et l'heure de la prise des repas étaient non seulement directement proportionnelles à la saison, au type de travail et à la santé du moine, mais elles étaient aussi liées au contexte géographique des monastères dispersés dans le territoire ou

${ }^{1}$ G. ANDENNA, Monasteri altomedievali nell'area subalpina e retica (secoli VIII-IX), in G. SPINELLI osb (dir.), Il monachesimo italiano dall'età longobarda all'età ottoniana (Secoli VIII-X), Atti del VII Convegno di studi storici sull'Italia benedettina (Nonantola, 10-13 settembre 2003), Cesena, 2006, p. 193-213.

${ }^{2} \mathrm{G}$. PENCO, Condizioni e correnti del monachesimo in Italia nel secolo VI, in Benedictina, 27, 1980, p. 93-95.

${ }^{3}$ G. ANDENNA, Monasteri..., op. cit. (n. 1), p. 193-194.

${ }_{4}^{4}$ A. de VOGUË, "Discretione precipuam". A quoi Grégoire pensait-il ?, in Benedictina, 22, 1975, p. 325-327.

${ }^{5}$ S. PRICOCO, La regola di S. Benedetto e le regole dei Padri, Verona, 1995, p. 99.

${ }^{6}$ A. DE VOGUË, Travail et alimentation dans les Règles de saint Benoît et du Maître, in Benedictina, 74, 1964, p. 242-251 ; B. STEIDLE, "Ante unam horam reflectionis", Roma, 1957, p. 73-104 ; G. ARCHETTI, "Nihil operi Dei praeponatur". Il tempo dei monaci nel Medioevo, in G. Archetti e A. Baronio (éd.), “Tempus mundi umbra aevi". Tempo e cultura del tempo tra Medioevo e età moderna, Atti dell'incontro nazionale di studio (Brescia, 29-30 marzo 2007), Brescia, 2008 (Storia, cultura e società, 1), p. 51-80. 
enfermés dans les murailles urbaines, situés sur les littoraux ou au centre des Apennins, près ou loin des lieux d'échange principaux.

Trois normes alimentaires - mesure de la nourriture, mesure de la boisson et heure des repas - sont fixées au tout début de la Règle 7 . Les règlements des ordres plus anciens attachaient au contraire moins d'importance aux institutions et au déroulement précis de la vie monastique ${ }^{8}$. Ils tendaient plutôt à énumérer les vertus à travers lesquelles le moine cherchait à réaliser son ascèse spirituelle : humilité, charité, maîtrise du corps et abnégation?.

Les objectifs de cette contribution sont pluriels. Il s'agit d'abord pour nous de mettre en relation les aspects liés au régime des moines, documentés par l'étude des vestiges archéologiques de plusieurs monastères du haut Moyen Âge, choisis comme échantillons-témoins dans le centre-nord de l'Italie. Nous tenterons de vérifier l'existence de rapports entre la vaisselle retrouvée dans ces cénobes et les habitudes alimentaires monastiques - afin de comprendre combien des préceptes établis par la Règle de saint Benoît étaient appliqués et respectés dans la pratique quotidienne. Nous examinerons ensuite la qualité et les caractéristiques de la culture matérielle de ces complexes religieux, pour comprendre si et dans quelle mesure les monastères peuvent avoir été aussi des "centres de pouvoir", de contrôle ou de médiation d'activités économiques importantes. Nous essayerons de définir enfin combien la lecture des documents alto-médiévaux peut influencer la reconstruction du paysage dans lequel le contexte monastique s'est implanté, en admettant que les aspects religieux, du moins à partir de la fin du VII e-début du VIII siècle, n'étaient que le "masque" derrière lequel s'opéraient les choix stratégiques des élites dominantes.

\section{LE RÉGIME ALIMENTAIRE DANS LA RÈGLE BÉNÉDICTINE}

Tous les monastères étaient dotés de règles propres et chaque abbé fondateur fournissait aux moines sa Règle. Ces principes s'inspiraient d'un type de monachisme fermé, basé sur la fuga mundi et la recherche d'un isolement volontaire, ou bien d'un style de vie plus ouvert comportant des rapports directs avec la société. Dans leur organisation, les premières expériences monastiques occidentales s'adaptèrent au régime cénobitique, même si certaines adoptèrent des formes mixtes ${ }^{10}$.

La finalité principale de la Règle de saint Benoît était d'organiser la vie cénobitique. La préoccupation d'assurer un équilibre alimentaire et la volonté de contrôler de fait la vie spirituelle des moines à travers les choix alimentaires, devaient les aider à atteindre la perfection de leur consécration à Dieu ${ }^{11}$. Un second aspect, les éloignait presque symboliquement de la table, les différenciant des habitudes des laïcs en pratiquant la modération et le renoncement ${ }^{12}$. Il y a lieu de garder à l'esprit qu'une partie des moines étaient issus de l'aristocratie du haut Moyen Âge. L'abondance de nourriture était alors considérée comme un principe fondamental de distinction sociale. Or presque tous les monastères étaient dotés de nombreuses propriétés foncières, de sorte que l'alimentation des moines aurait pu être aussi variée et copieuse que celle de leurs parents laïcs. Le fait d'imposer des repas sobres était donc significatif d'un choix de vie précis, qui imposait des sacrifices et des renoncements ${ }^{13}$. Les chapitres XXXVIII et XXXIX de la Règle évoquent expressément des dispositifs alimentaires, suivis du chapitre XL qui traite de la qualité des aliments et de la quantité de vin autorisée ${ }^{14}$.

Le contrôle alimentaire des moines, et pas seulement bénédictins, fut l'un des choix établis pour favoriser l'ascétisme, peut-être plus encore que les prières de la liturgie des heures ${ }^{15}$. Cette prédilection est accentuée par le fait que nombre de moines provenaient d'un milieu social élevé, non opprimé par une éventuelle pénurie vivrière. Ils aimaient la bonne chère ${ }^{16}$. Dans le cas contraire, ils n'auraient pas eu besoin d'invitations constantes et répétées à la modération et au renoncement des aliments considérés comme les plus désirables, qui étaient également les plus coûteux, tels que les épices et les aliments riches en significations symboliques fortes, comme la viande rouge ${ }^{17}$. Les habitudes alimentaires proposées par la Règle bénédictine consistaient, quoi qu'il en soit, en un régime sain et naturel, plutôt monotone, conditionné en outre par la production agricole saisonnière et par les différences de localisation entre les monastères ${ }^{18}$.

\footnotetext{
7 V. FATTORINI, L'alimentazione nella Regola di San Benedetto, Milano, 1995 ; G. ARCHETTI, "Mensura victus constituere". Il cibo dei monaci tra Oriente ed Occidente, in L'alimentazione nell'alto medioevo: pratiche, simboli, ideologie, Sessantatreesima settimana di studio (Spoleto, 9-14 aprile 2015), Spoleto, 2016, p. 757-797.

${ }^{8}$ H.-I. MARROU, S. Agostino e la fine della cultura antica, Milano, 1971, p. 183-187.

${ }^{9}$ G. PENCO, Storia della Chiesa in Italia, Milano, 1978, p. 65-67.

${ }^{10} \mathrm{G}$. TURBESSI, La Regola di San Benedetto nel contesto delle antiche regole monastiche, Roma, 1972.

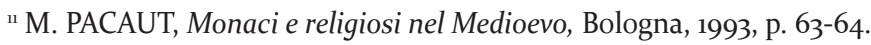

${ }^{12}$ P. LUGANO, San Benedetto. Vita e Regola, Roma, 1929, p. 97-104.

${ }^{13}$ L. SALVATORELLI, San Benedetto e l'Italia del suo tempo, Bari, 1929, p. 38-41.

${ }^{14}$ A. LENTINI, La Regola di San Benedetto di Montecassino, Montecassino, 1994, p. 43-47. Sur le thème du vin dans la Règle, cf. G. ARCHETTI, "De mensura potus". Il vino dei monaci nel medioevo, in ID. (éd.), La civiltà del vino. Fonti, temi e produzioni vitivinicole dal Medioevo al Novecento, Atti della VII Biennale di Franciacorta (Monticelli Brusati-Antica Fratta, 5-6 ottobre 2001), Brescia, 2003, p. 205-326 ; ID., "Infundit vinum et oleum". Olio e vino nella tradizione monastica, in Olio e vino nell'alto medioevo, Settimane di studio della Fondazione Centro italiano di studi sull'alto medioevo, LIV, Spoleto, 2007, p. 10991209 ; ID., "Il vino non è per i monaci". Appunti sparsi sugli usi monastici antichi, in F. MONTELEONE \& L. LOFOCO (éd.), "Dulcius nil est mihi veritate". Studi in onore di Pasquale Corsi, Foggia, 2015, p. 65-87.

${ }^{15} \mathrm{G}$. PENCO, Storia del monachesimo in Italia dalle origini alla fine del Medioevo, Roma, 1961, p. 75-84.

${ }^{16}$ J. LECLERCQ, Umanesimo e cultura monastica, Milano, 1989, p. 174-175.

${ }^{17}$ S. PRICOCO, L'isola dei santi. Il cenobio di Lerino e le origini del monachesimo gallico, Roma, 1978, p. 621-641.

${ }^{18}$ E. CIRELLI, La dieta dei monaci. Cultura materiale e alimentazione nei monasteri benedettini tra IX e X secolo, in HAM, 19, 2013 , p. $227-228$.
} 


\section{DOCUMENTS HISTORIQUES ET SOURCES ARCHÉOLOGIQUES DANS L'ÉTUDE DES IMPLANTATIONS MONASTIQUES DE L'ANTIQUITÉ TARDIVE ET DU HAUT MOYEN ÂGE : LE CROISEMENT DES DONNÉES}

D'une étendue limitée, les îles ligures - Gallinara et Bergeggi dans les eaux de la mer Tyrrhénienne occidentale, Tino et Tinetto dans celles de la mer Tyrrhénienne orientale
- représentèrent dès la période ancienne des points faciles d'ancrage pour les bateaux faisant route vers la Gaule et l'Espagne, du fait de leur position géographique à proximité de la côte. Bien qu'une fréquentation presque continue, certes sporadique et éphémère, soit attestée à partir des siècles romains, il est très difficile dans ces contextes de définir le type d'occupation, sur la base du matériel retrouvé et en l'absence de structures stables ${ }^{19}$. Si les sources, à commencer par

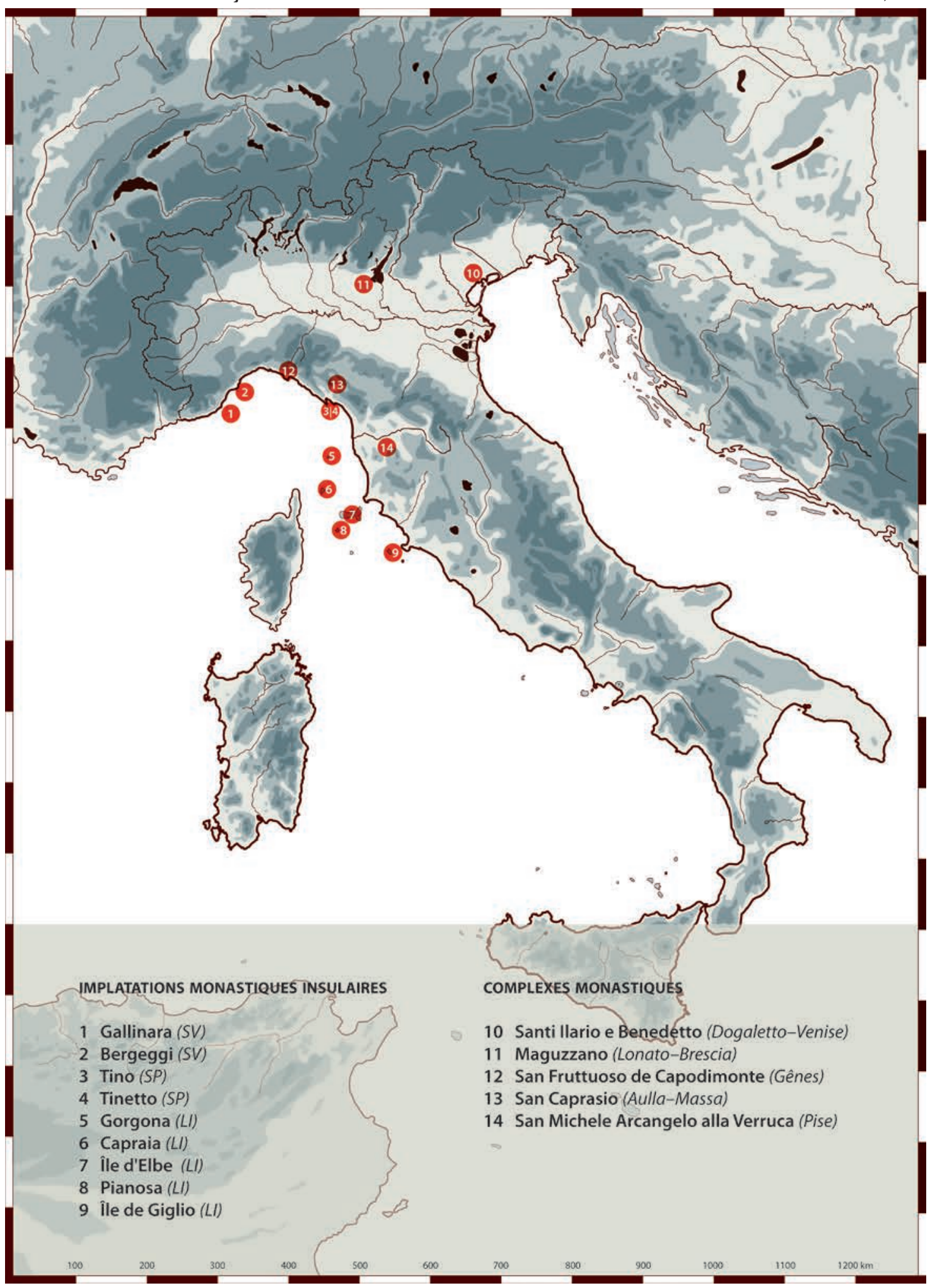

Figure 1. Localisation de tous les sites monastiques examinés (C Rossana Managlia)

${ }_{19}$ P. PERGOLA, B. MAZZEI, F. SEVERINI, L'implantation chrétienne dans les îles mineures des archipels toscan et ligure (Antiquité tardive et haut Moyen Âge), in M. PASQUALINI, P. ARNAUD, C. VARALDO (dir.), Des îles côte à côte. Histoire du peuplement des îles de l'Antiquité au Moyen Âge (Provence, Alpes-Maritimes, Ligurie, Toscane), Actes de la table ronde de Bordighera (12-13 décembre 1997), Aix-en-Provence-Bordighera, 2003, p. 193-204 (Supplément au Bulletin Archéologique de Provence, 1). 
saint Ambroise, attestent combien étaient nombreux ceux qui choisirent les îles pour s'éloigner du monde et mener une vie de prière, de méditation dans la pauvreté, nous ne disposons pas de preuves directes de la vie cénobitique sur ces îles aux premiers siècles de la chrétienté ${ }^{\circ}$ (fig. 1).

La présence d'installations monastiques insulaires parfaitement organisées au Moyen Âge et les traditions locales qui font remonter ces monastères, souvent liés au séjour ou au lieu de sépulture d'un personnage vénéré, à l'Antiquité Tardive, rendent plausible la théorie de l'antiquité présumée de telles implantations, mais elles n'ont pas toujours été confirmées par des données archéologiques dignes de foi. Cela vaut notamment pour l'île de Gallinara. En revanche, on a effectivement trouvé à Bergeggi de la vaisselle de l'Antiquité Tardive, qui confirmerait la fréquentation d'un édifice cultuel des $\mathrm{V}^{\mathrm{e}}-\mathrm{VI}^{\mathrm{e}}$ siècles, que l'on lie à la présence de saint Eugène par rapport au monastère du $\mathrm{X}^{\mathrm{e}}$ siècle. L'absence de structures concomitantes de l'église la plus ancienne laisse pourtant planer le doute sur la présence effective d'une communauté monastique stable avant le $\mathrm{X}^{\mathrm{e}}$ siècle ${ }^{21}$.

Les deux îles du Tino et du Tinetto, positionnées devant le promontoire de Portovenere, sont quant à elles bien liées au culte de saint Vénère. Dans le premier cas, l'implantation monastique stable n'est documentée qu'à partir du milieu du XI ${ }^{\mathrm{e}}$ siècle, ce qu'ont confirmé les investigations archéologiques effectuées dans le complexe médiéval d'une des abbayes bénédictines les plus célèbres de la Ligurie ${ }^{22}$. Le matériel récupéré dans les fouilles du cloître roman prouve que l'île du Tino était occupée à l'époque impériale centrale et tardive, suivie par une implantation stable, plutôt pauvre, aux $\mathrm{V}^{\mathrm{e}}$ et $\mathrm{VI}^{\mathrm{e}}$ siècles, alors même que les attestations de fréquentation sont peu nombreuses pour les $\mathrm{VII}^{\mathrm{e}}-\mathrm{X}^{\mathrm{e}}$ siècles, période à laquelle les sources situent le plus ancien bâtiment cultuel $^{23}$.

On a découvert sur le proche îlot du Tinetto les vestiges d'un complexe du Moyen Âge, lié au culte de saint Vénère. Bien que l'on y ait reconnu différentes phases entre le $\mathrm{XI}^{\mathrm{e}}$ et le XIII ${ }^{\mathrm{e}}$ siècle, les données archéologiques n’ont pas fourni assez d'éléments pour établir la destination de l'ensemble, même s'il est probable qu'il s'agissait à l'origine d'un lieu de culte lié à la présence de reliques, successivement transformé en un sanctuaire doté des structures susceptibles de recevoir des pèlerins, à moins que ce ne soit un ermitage temporaire utilisé par les moines de l'île du Tino ${ }^{24}$. Bien que certains éléments publiés soient plutôt faibles, la présence sur les îles ligures de bâtiments de culte tardo-antiques liés au passage (Martin) ou à la sépulture d'un saint vénéré
(Eugène et Vénère) semble avérée. Prouver l'existence d'une communauté monastique précoce est plus compliqué. La céramique confirme la présence humaine sur les îles dans l'Antiquité Tardive, mais seul un cas, celui de l'île du Tino, offre une continuité de fréquentation du $\mathrm{IV}^{\mathrm{e}}$ au VII ${ }^{\mathrm{e}}$ siècle ${ }^{25}$. Ces éléments pourraient être reliés à des formes embryonnaires de vie cénobitique, encore imparfaitement organisées. L'absence de structures monastiques stables se justifierait alors par le type de vie plus ou moins solitaire et précaire, que les religieux auraient choisi de mener ${ }^{26}$.

Quant aux îles de l'archipel toscan, nombreux sont les auteurs qui ont laissé d'importants témoignages directs ou indirects sur le phénomène monastique (Augustin, Rutilius Namatianus, Jérôme et Grégoire le Grand). Cependant, si la consistance de la documentation littéraire représente une preuve objective de la présence de formes de vie cénobitique, leur diffusion, leur extension, leur continuité et leur évolution dans le temps doivent encore être confirmées par d'autres sources, à commencer par l'archéologie ${ }^{27}$.

Une différence profonde apparaît entre données textuelles et archéologiques pour ce qui est de l'île de Gorgone. Les documents font des références assurées et extraordinairement riches à des monasteria, qu'il faut entendre chez Grégoire le Grand comme des lieux de retraite spirituelle et de solitude, mais rien ne le confirme pour l'instant d'un point de vue archéologique ${ }^{28}$. Ce n'est qu'en 1051 qu'un privilège pontifical apporte la première attestation d'un monastère d’observance bénédictine, administré par un abbé répondant au nom de Bonus ${ }^{29}$. L'île de Capraia présente le même déséquilibre entre sources littéraires et matérielles, probablement même d'une façon plus marquée. Un monastère fondé au Moyen Âge est traditionnellement situé au lieu-dit La Piana, à proximité de l'église Saint-Étienne qui remonte au XIII ${ }^{\mathrm{e}}$ siècle. Dans les environs, on a observé des cuves rupestres, interprétées comme des installations de foulage du raisin, mais aucune investigation n'a été menée pour déceler la présence d'implantations antérieures. Quant à l'île d'Elbe le seul témoignage concerne Cerbone, le saint évêque de Populonia, qu'évoque Grégoire le Grand dans ses Dialogues ${ }^{30}$. Quelques structures maçonnées encore partiellement visibles sur le mont Capanne, pourraient correspondre à une petite implantation monastique du VI siècle, mais aucune vérification archéologique n'a encore précisé les choses ${ }^{31}$. On dispose pour l'île de Pianosa d'un unique document des premières décennies du XII ${ }^{\mathrm{e}}$ siècle ${ }^{32}$; le pape Gélase II (1118-1119) y réaffirme la possession de Pianosa par l'abbaye S. Salvatore e S. Mamiliano, fondée au XI

\footnotetext{
${ }^{20}$ Ibid., p. 197.

${ }^{21}$ Ibid., p. 198.

${ }^{22}$ Ibid., p. 198-199.
}

${ }^{23}$ A. FRONDONI, Tino isola, in ID. (éd.), Archeologia cristiana in Liguria. Aree ed edifici di culto tra IV e XI secolo, Atti VIII Congresso Nazionale di Archeologia Cristiana (Genova-Sarzana-Finale Ligure-Albenga-Ventimiglia, 21-26 settembre 1998), Bordighera, 2001, cartes 25-1/2.

${ }^{24}$ Ibid., carte 26/1.

${ }^{25}$ G. PENCO, Storia del monachesimo..., op. cit. (n. 15).

${ }^{26}$ P. PERGOLA, B. MAZZEI, F. SEVERINI, L'implantation chrétienne..., op. cit. (n. 19), p. 199.

${ }^{27}$ Ibid., p. 200.

${ }^{28}$ Gregorii I papae, Registrum Epistolorum, I. 50 = Corpus Christianorum, 1. 140, 63-64.

${ }^{29}$ P. PERGOLA, B. MAZZEI, F. SEVERINI, L’implantation chrétienne..., op. cit. (n. 19), p. 200.

$3^{30}$ Gregorii I papa, Dialogi, 3.11 = Patrologia latina, 77, 237-240.

${ }^{31}$ P. PERGOLA, B. MAZZEI, F. SEVERINI, L'implantation chrétienne..., op. cit. (n. 19), p. 201.

${ }^{32}$ G. BARTOLOZZI CASTI, La catacomba della Pianosa. Stato della Ricerca, in Pianosa: passato, presente e futuro, Atti del Convegno di Studi (Isola di Pianosa, 17-19 maggio 1997), Portoferraio, 1997, p. 3-16. 


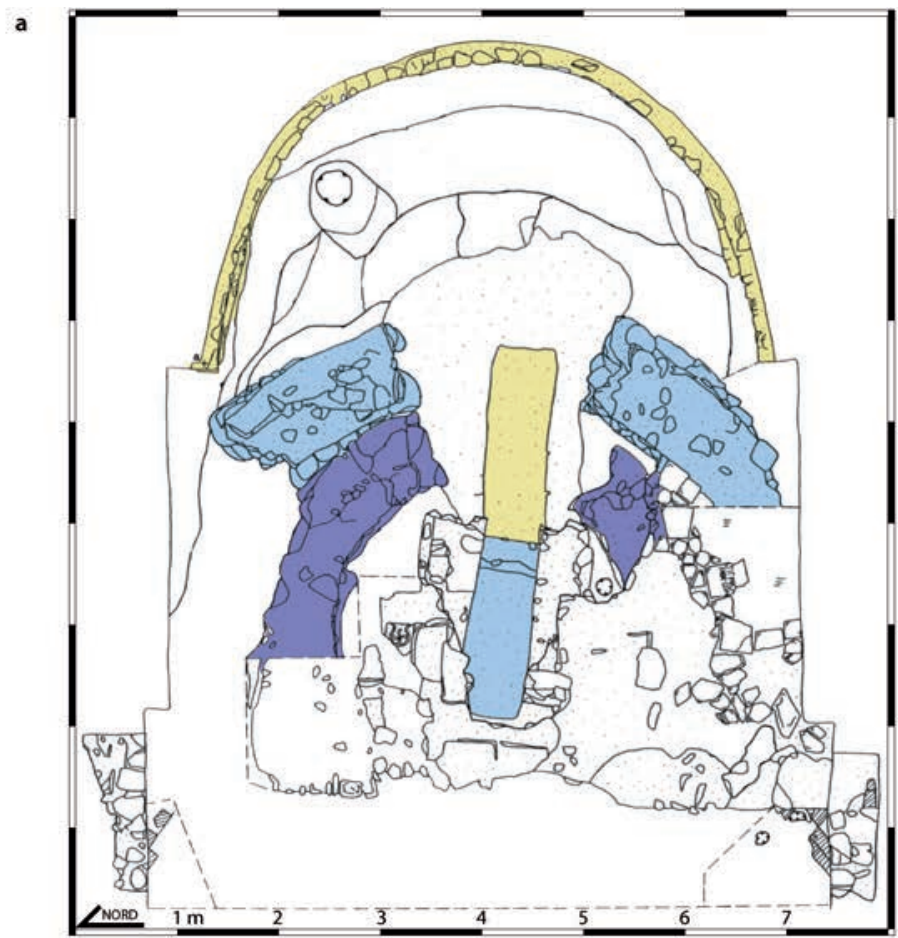

b
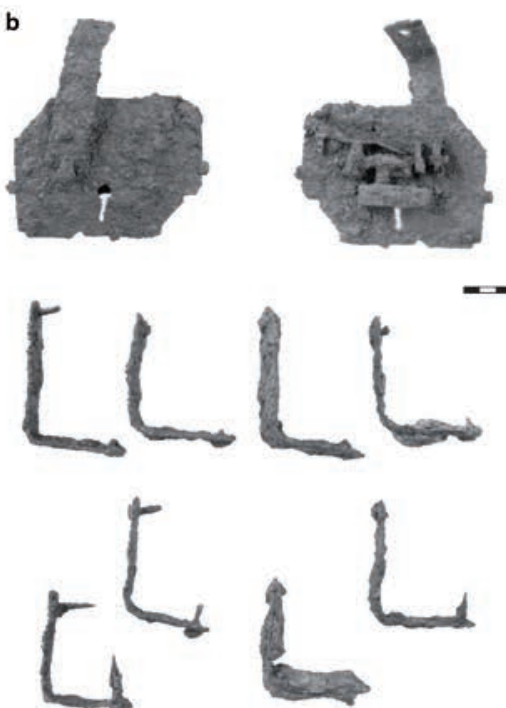

Figure 2. S. Caprasio (MS) - a. Plan de la zone de l'abside en fin de fouille; b. Plaques de serrure, cornières et gonds recueillis dans le sépulcre alto-médiéval (d'après E. ARSLAN et alii, Indagini..., op. cit. [n. 36], fig. 6, p. 174, fig. 13, p. 179, modifiées)

siècle par les bénédictins sur l'île de Montecristo. Quant à celle de Giglio, bien que les sources littéraires signalent une implantation monastique à la fin $\mathrm{du} \mathrm{V}^{\mathrm{e}}$ siècle, aucune donnée archéologique ne vient la corroborer ${ }^{33}$. Enfin, on ignore tout d'une quelconque installation monastique remontant à l'Antiquité Tardive sur l'île de Giannutrij3.

L'examen comparé des sources littéraires et archéologiques offre bien peu d'éléments convergents pour préciser l'établissement effectif de communautés de moines dans l'Antiquité Tardive sur les îles toscanes. Sur Gorgone et
Montecristo, la documentation littéraire permet seule de supposer des formes de vie érémitiques, difficilement perceptibles par le biais de l'archéologie 35 .

Le 27 mai 884, le notaire Pietro entérina à Lucques la volonté d'Adalbert I Ier de Toscane de bâtir une église à Lunigiane, au confluent du fleuve Magra et du torrent Aulella, à l'usage d'une abbaye déjà constituée où les moines ne s'étaient pas encore installés, et qui deviendra entre la fin du $\mathrm{IX}^{\mathrm{e}}$ et le XII ${ }^{\mathrm{e}}$ siècle le monastère de $\mathrm{S}$. Caprasio d'Aulla. Ainsi était planifiée une implantation protégée, susceptible de répondre à la conjoncture économique et à un besoin accru de sécurité, dans la période d'instabilité politique que caractérise traditionnellement le passage de la phase post-carolingienne au $\mathrm{X}^{\mathrm{e}}$ siècle. Après ce document constitutif, le premier texte concernant l'histoire du monastère est un diplôme de 1077, où il apparaît sous le nom d'Abbazia Santi Caprasi ${ }^{36}$.

Probablement érigée au cours du VIII ${ }^{\mathrm{e}}$ siècle, l'église la plus ancienne, à abside unique, possédait un mobilier liturgique de grande qualité. À ce premier bâtiment succéda un deuxième, qui réutilisa une partie du mobilier sculpté précédent, lui ajoutant un nouveau ciborium ou un autre élément qui portait une inscription évoquant la fondation du complexe. Dans le même secteur de l'abside, une fosse creusée sous l'autel a révélé une caisse-reliquaire en bois, dont sont conservés les fixations en fer (16 cornières, deux charnières, une serrure et quatre clous) et quelques fragments osseux, datés par le radiocarbone entre 410 et $560^{37}$ (fig. 2).

Autour du milieu du $\mathrm{XI}^{\mathrm{e}}$ siècle, une église à trois nefs vint englober les structures précédentes. On exhuma alors les os du sépulcre antérieur pour les déposer dans un nouveau sarcophage-reliquaire en stuc, de forme rectangulaire, que l'on inséra au milieu de l'abside principale, dans une fosse soigneusement remplie de sable fin et scellée par des plaques de marbre en remploi. Au-dessus, fut posée une couverture en bâtière faite de claveaux en pierre spécialement profilés, sur laquelle on accumula des galets liés au mortier, qui formèrent le socle du nouvel autel. Tout l'espace autour de l'église fut dévolu à l'ample cimetière né au haut Moyen Âge et qui fonctionna jusqu'à la période moderne. La conservation partielle des sépultures les plus anciennes empêche de proposer des considérations précises sur les modalités d'inhumation,

33 P. PERGOLA, B. MAZZEI, F. SEVERINI, L’implantation chrétienne..., op. cit. (n. 19), p. 202.

34 Ibid., p. 202-203.

35 Ibid., p. 203.

$3^{6}$ E. ARSLAN, F. BARTOLI, R. BOGGI, L. BURDASSI, M. L. CASATI, E. GIANNICHEDDA, R. LANZA, B. LIPPI, F. MALLEGNI, G. MENNELLA, G. PAGNI, O. RATTI \& T. MANNONI, Indagini archeologiche nella chiesa dell'abbazia altomedievale di San Caprasio ad Aulla (MS), Firenze, 2006 (Archeologia medievale, 33), p. 167-168.

37 Ibid., p. 177-180. 

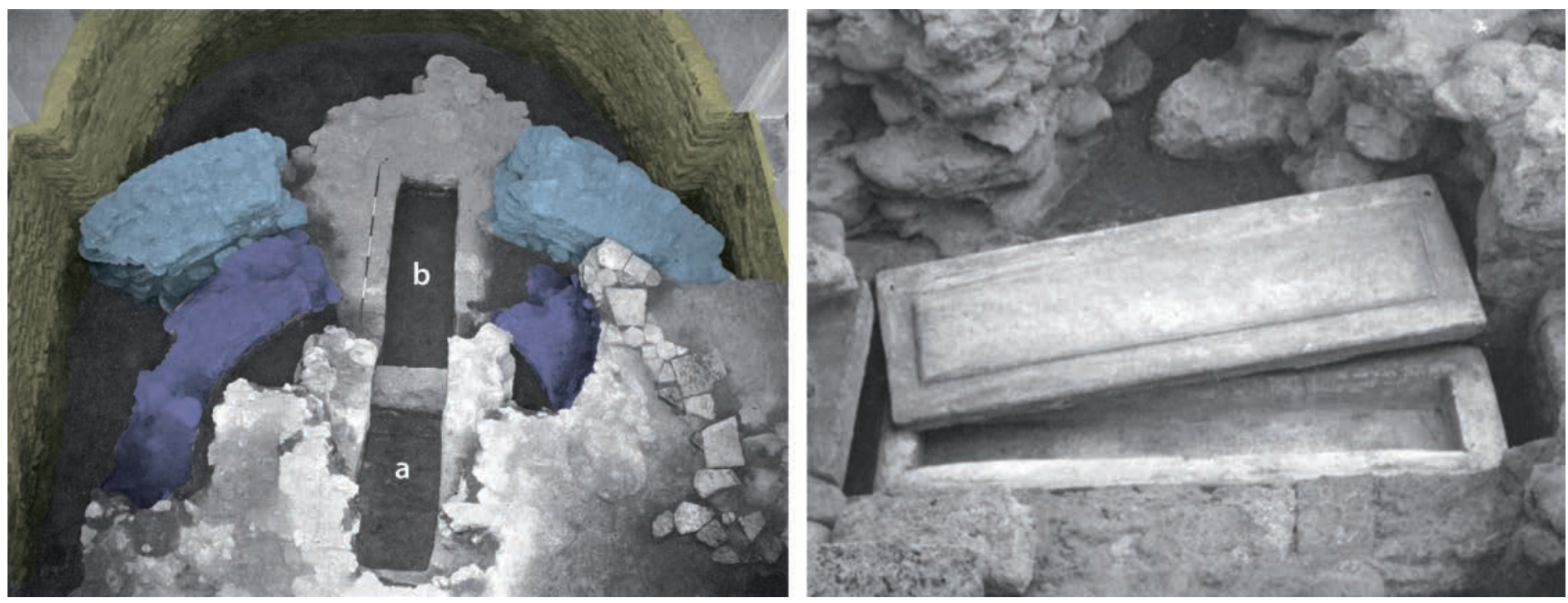

Figure 3. S. Caprasio (MS) - a-c. Absides des phases du haut Moyen Âge (a-b) et de l'église médiévale (c); d-e. Fosses des sépulcres privilégiés destiné à la caisse primitive en bois (d) puis au sarcophage (e). Sarcophage-reliquaire en stuc (d'après E. ARSLAN et alii, Indagini..., op. cit. [n. 36], fig. 9, p. 174, fig. 17, p. 175, modifiées)

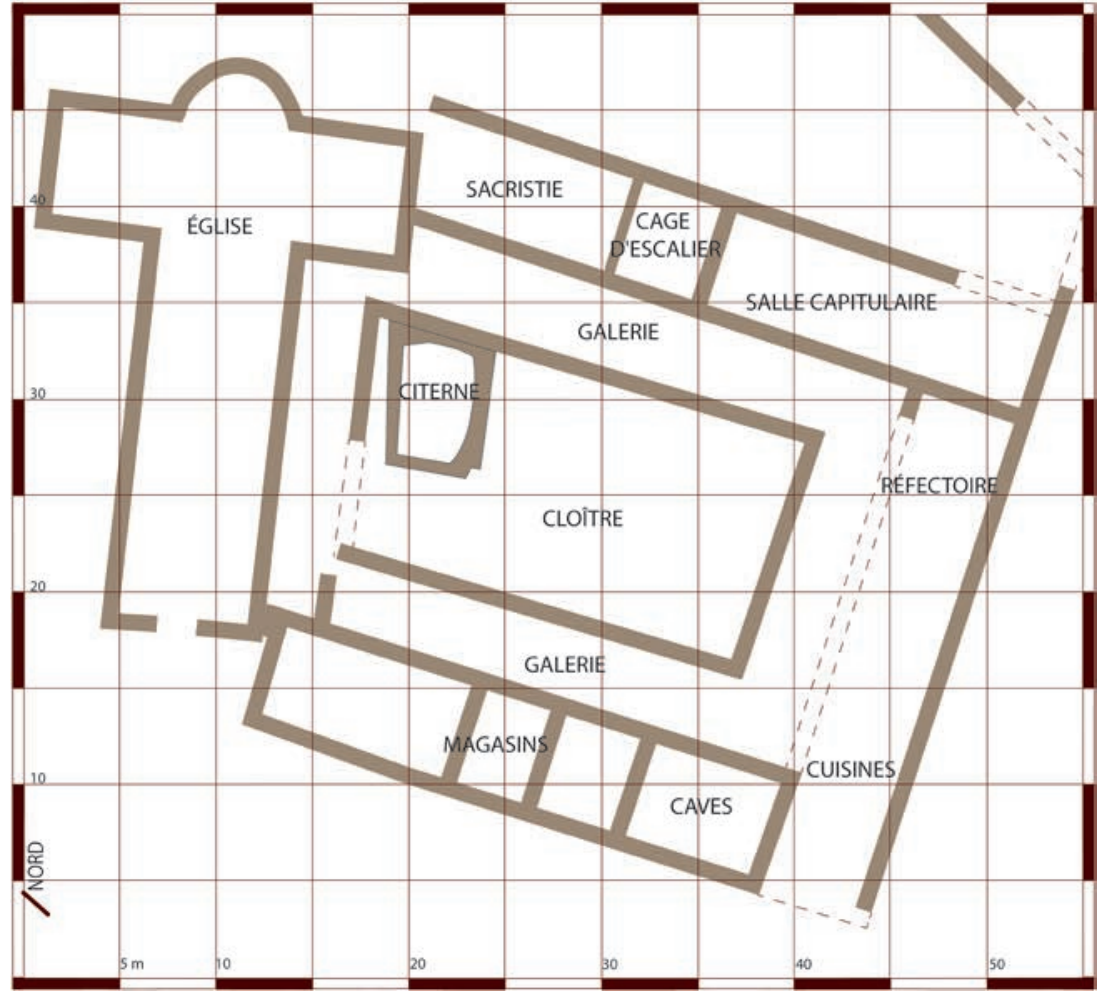

Figure 4. S. Michele alla Verruca (PI), plan général (d'après S. GELICHI, A. ALBERTI, M. $D A D \grave{A}$, L'indagine archeologica..., op. cit. [n. 47], fig. 3, p. 66, modifiée)

même s'il s'agit toujours d'individus adultes aux membres supérieurs alignées le long des hanches $3^{8}$ (fig. 3).

Il est évidemment impossible de proposer une corrélation directe entre les squelettes des tombes des $\mathrm{V}^{\mathrm{e}}$-VI $\mathrm{e}$ siècles et les restes osseux de saint Caprais - à qui la tradition locale attribue une partie de la christianisation des zones francoprovençales, où il serait décédé en 433 - dont les reliques ont localement fait l'objet d'un culte pendant au moins 1100 ans. Du point de vue archéologique, ces os fournissent des données fondamentales pour étudier et comprendre un phénomène complexe qui caractérisa le monachisme alto-médiéval. Ils irradiaient d'une composante "spirituelle", la virtus, qui conduisit des milliers de personnes à se rendre là où des dépouilles mortelles de saints présumés étaient conservées, mais ils offraient aussi un ressort "économique" en mesure de concentrer d'importantes ressources autour de ces déplacements ${ }^{39}$.

$\mathrm{Au}$ Moyen Âge, S. Michele Arcangelo alla Verruca était l'un des monastères bénédictins les plus importants des Monti Pisani, parmi les fondations développées entre les trois dernières décennies du $\mathrm{X}^{\mathrm{e}}$ siècle et les années 106o. D’abord propriété du comte Féruald Aldobrandeschi puis de l'évêché lucquois, l'église S. Angelo alla Verruca apparaît dans un document du 30 juin 861. Eriprand, fils d'Ildiprand, y dédommageait l'évêque de Lucques Jérémie à l'aide de sa part de propriété dans l'église Saint-Benoît de Settimo, pour solde du déguerpissement de l'« ecclesia et rebus meis illis in locho Verruchula, chuius vocabuli est beati S. Angeli, cum omni adiacentia et pertinentia sua », qu'Eriprand avait reçue de son ancêtre maternel Féruald et qu'il avait échangée précédemment avec le prédécesseur de Jérémie, Bérenger, contre quelques biens situés près de Roselle, ${ }^{40}$ (fig. 4).

Dans la deuxième moitié du $\mathrm{X}^{\mathrm{e}}$ siècle l'église demeura sous le contrôle de l'évêché de Lucques. Le 6 août 913 elle fut cédée en location par l'évêque Pierre à un certain Aripert et à ses enfants Auripert et Grimoald diacre, contre un cens annuel de 12 deniers d'argent à payer au mois d'août, avec l'obligation d'y tenir des offices ainsi que des « luminaria et incensum $»^{41}$. La typologie du bien semble alors se rapporter à une structure plus complexe que la précédente, avec la mention de quelques constructions liées à l" « Ecclesia illa

\footnotetext{
${ }^{8}$ Ibid., p. 180-182.

39 Ibid., p. 172-175.

${ }^{40}$ G. GIULIANI, Il monastero di San Michele alla Verruca: profilo delle vicende storiche, in S. GELICHI \& A. ALBERTI (éd.), L'aratro e il calamo. Benedettini e Cistercensi sul Monte Pisano. Dieci anni di archeologia a San Michele alla Verruca, Pisa, 2005, p. 11.

${ }^{41}$ Ibid., p. 13.
} 
cujus vochabulum est beatissimi Sancti Angeli, sita locho ubi vocitatur Verruchula, qui est pertinentis suprascripti episcopatui vestri sancti Martini, jamdicta Domini ecclesia Sancti Angeli cum fundamento, et omnen edificio suo $»^{42}$.

La première attestation d'une église et d'un monastère de Saint-Michel Archange « sito loco et finibus, ubi dicitur Verruca, que est de sub regimine et potestatem ecclesiae episcopatui nostri Sancti Martini [...] cum fundamento et omne edificio suo » se trouve dans un document du 4 mai 996, nommant Gérard, évêque de Lucques, comme bénéficiaire du versement de la redevance annuelle de huit sous au mois de janvier ${ }^{43}$. Ce document ainsi qu'un diplôme impérial du 21 juillet 996, par lequel Otton III concédait « interventu ac petitione Hugonis marchionis » à l'abbaye de Sesto « roccha [...] quae dicitur Verrucha, cum omnibus sibi pertinentibus rebus » peuvent être évalués dans le contexte d'une expansion des intérêts du monastère de S. Salvatore de Sesto vers le val d'Arno pisan, en créant un patrimoine territorial de dimensions correctes dans cette zone d'importance stratégique ${ }^{44}$.

Les rapports avec S. Salvatore se croisent dans les premiers temps du monastère de la Verruca et se maintiennent au moins jusqu'à la fin du XI ${ }^{\mathrm{e}}$ siècle. En 1097, le prieur de $\mathrm{S}$. Michele Bernard est attesté seul, après presque un siècle où les deux abbés figuraient toujours ensemble dans les documents ${ }^{45}$. Les privilèges impériaux d'Henri II le 25 avril 1020, de Conrad II le 6 avril 1027 et d'Henri III le 4 juillet 1053 confirment tous à l'abbaye de Sesto la possession de la "forteresse de la Verruca" ${ }^{6}$.

Au niveau archéologique, si la première phase de construction du monastère peut être datée entre la fin du $\mathrm{X}^{\mathrm{e}}$ et le $\mathrm{XI}^{\mathrm{e}}$ siècle, le peu qui subsiste ne suffit pas à définir la fonction des structures, mais on constate l'absence d'organisation planifiée de l'espace monastique ${ }^{47}$. Il reste à éclaircir l'implication des commanditaires (le prieur de Sesto ?) sur le chantier de construction primitif de S. Michele, en considérant qu'à la même période on a investi à S. Salvatore dans des travaux de construction aboutissant à une restructuration complète $^{48}$. Cette hypothèse supposant la présence d'ouvriers spécialisés lucquois, que seules de riches institutions comme pouvait l'être à l'époque l'abbaye de Sesto pouvaient employer, semble recevable : les techniques de maçonnerie de cette première phase sont en effet comparables avec des maçonneries contemporaines mises à jour dans des fouilles urbaines à Lucques ${ }^{49}$.
Le manque d'une charta dotis ou d'un acte de fondation, perdu ou n'ayant même jamais existé, nous impose de réfléchir au rôle que joua le marquis Ugo de Tuscie (970-1001) dans cette phase de fondation-rénovation-reconversion de l'église de S. Angelo au monastère de S. Michele. Il désirait sans doute renforcer les monastères impériaux, en les transformant en centres d'organisation pour les biens fiscaux dispersés à la fin du $\mathrm{X}^{\mathrm{e}}$ siècle, une période confrontée à une situation institutionnelle en évolution rapide, principalement du fait de la conquête du pouvoir par les comites. Une solution consista à faire contrôler les territoires privés et les biens d'origine publique, en investissant de cette responsabilité les organismes ecclésiastiques. Le marquis put ainsi exercer son pouvoir sans présence effective ${ }^{50}$.

La première attestation textuelle du monastère de $\mathrm{S}$. Fruttuoso de Capodimonte, situé à Camogli, à proximité de Gênes, date du $1^{\text {er }}$ septembre 977, lorsque Amalbert, fils de Dodo da Gremiasco fit don au monastère de deux « mansi in loco et fundo nominatum Selva ». Entre 994 et 999 à ces biens s'en ajoutèrent d'autres, localisés sur le même promontoire de Portofino ou dans la partie septentrionale du Val Borbera et à Casalcermelli dans le Piémont méridional, qui étaient le fruit des donations d'aristocrates locaux et de l'impératrice Adélaïde de Bourgogne ${ }^{51}$.

Dans l'ensemble la typologie des biens et les revenus éventuels pouvant être assurés ne sont pas très importants - assez pour subvenir aux besoins d'une communauté cénobitique, mais trop peu pour financer des opérations de construction ou de rénovation. Il y a donc lieu de supposer l'intervention de mécènes dont les donations ne peuvent pas être datées antérieurement à la première moitié du XI ${ }^{e}$ siècle ${ }^{52}$. Rappelons aussi un document épiscopal, attribué à l'an 994, par lequel l'évêque Jean de Gênes confirmait au monastère de $\mathrm{S}$. Fruttuoso des biens à Capodimonte, en établissant, d'une façon implicite, sa reconnaissance ${ }^{53}$.

Les fouilles archéologiques à S. Fruttuoso de Capodimonte se sont principalement concentrées sur le cloître médiéval et les zones limitrophes, où a été documentée la première phase d'utilisation du site par une petite communauté. Sa présence est confirmée par les tessons recueillis dans des remblais de nivellement contre les maçonneries les plus anciennes. Ces fragments de vaisselle de table importée d'Afrique du Nord, de céramiques communes et de récipients de transport sont tous datables du VI ${ }^{\mathrm{e}}$ siècle ${ }^{54}$. Une seconde phase caractérise certaines portions de maçonneries, dans la

\footnotetext{
${ }^{2}$ Ibid., p. 14.

${ }^{43}$ Ibid., p. 14 .

${ }^{44}$ Ibid., p. 14.

45 Ibid., p. 14 .

${ }^{46}$ A. ALBERTI, I monasteri del Monte Pisano (X-XII secolo). Fondatori, committenti e gestione delle risorse, in L'aratro e il calamo..., op. cit. (n. 40), p. 35.

${ }^{47}$ S. GELICHI, A. ALBERTI, M. DADÀ, L’indagine archeologica del monastero di San Michele alla Verruca: la periodizzazione della sequenza insediativa, in L'aratro e il calamo..., op. cit. (n. 40), p. 63.

${ }^{48}$ A. ALBERTI, I monasteri..., op. cit. (n. 46), p. 36.

${ }^{49} \mathrm{Ibid} .$, p. 36.

${ }^{50}$ G. GIULIANI, Il monastero..., op. cit. (n. 40), p. 14.

${ }^{51}$ D. CALCAGNO \& M. CAVANA, Storia e architettura: note per lo studio del complesso di San Fruttuoso, in A. FRONDONI (éd.), Gli Stucchi di San Fruttuoso di Capodimonte, Genova, 2008, p. 33.

${ }^{52}$ Ibid., p. 33.

53 Ibid., p. 34

${ }^{54}$ A. GARDINI, Gli scavi della chiesa monastica di San Fruttuoso, in Gli Stucchi..., op. cit. (n. 51), p. 26.
} 


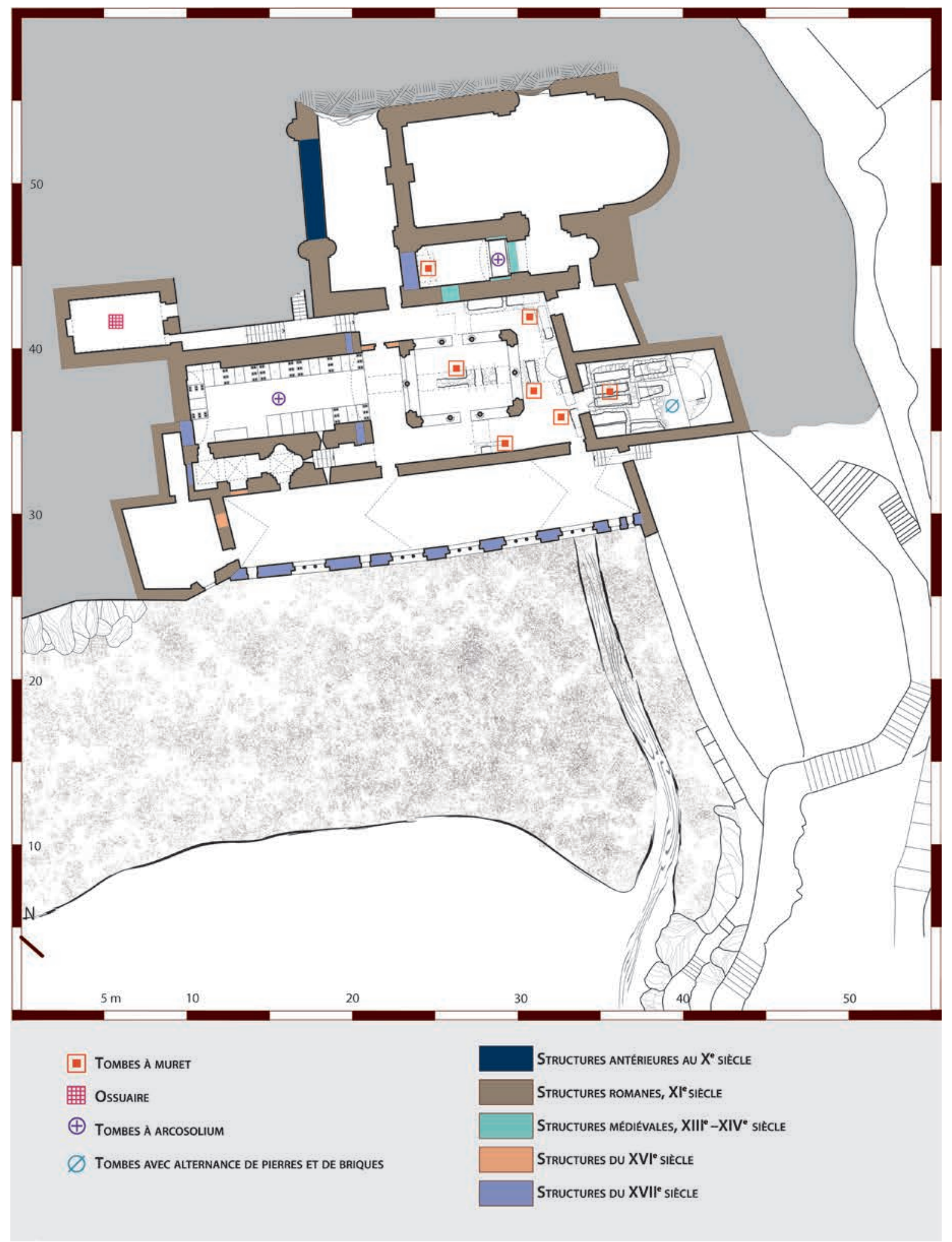

Figure 5. S. Fruttuoso de Capodimonte (GE), plan général avec phases de construction (d'après A. GARDINI, Gli scavi..., op. cit. [n. 54], fig. 29, p. 27, modifiée)

partie nord du complexe architectural et dans les fouilles de l'église, où l'on a repéré trois murs fermant une pièce qui correspondrait à un bâtiment conventuel primitif en usage à la fin du X $\mathrm{X}^{\mathrm{e}}$ siècle ${ }^{55}$. Les fouilles du noyau principal du complexe ont généré plus d'informations ; on y a reconnu l'état originel de l'église, au sol dallé en plaques d'ardoise et de pierre. Sa construction commença au début du XI ${ }^{\mathrm{e}}$ siècle et s'acheva entre le milieu et le dernier quart du même siècle, date étayée par la découverte d'une série de monnaies frappées à Pavie au-dessous du dallage. L'église était subdivisée en deux secteurs séparés par un mur (de chancel ?) portant une frise en stuc ; elle était bordée par une sacristie, alors qu'une pièce à abside devait correspondre à la salle capitulaire, qui communiquait avec une vaste pièce rectangulaire ${ }^{56}$ (fig. 5).

L'abbaye Santa Maria Assunta de Maguzzano se situe à proximité de la rive sud du lac de Garde, le long d'une

55 Ibid., p. 14-16

${ }^{56}$ Ibid., p. 12-13. 
voie traversant la via Postumia. La première attestation de l'abbatiola Magonziani apparaît dans un décret de l'évêque Rathier de Vérone daté autour de 966 : un particulier, qualifié de constructor, l'avait fondé, sans indication de date ${ }^{57}$. Ce décret épiscopal attirait l'attention sur l'état de dégradation du monastère, incendié par les Hongrois, et désavouait un individu, marié et père de famille, qui s'était autoproclamé abbé. Rathier décida de modifier le statut juridique du monastère, en maintenant le respect des règles cénobitiques " in memoriam antiquae consuetudinis in laudibus, matutinis, prima, tertia, sexta, nona, vespera et completorio ", et en rétablissant une communauté religieuse, mais séculière, composée de trois prêtres qui devaient célébrer les offices quotidiens, d'un diacre, d'un curé et de quelques clercs guidés par un presbyter honorabilis $5^{8}$.

Il reste à déterminer à quel moment les moines réguliers sont revenus au monastère. Dans sa donation effectuée en 1090, Ubert, fils du comte de Parme, transmettait la propriété d'une parcelle de terre arable à l'église $S$. Maria di Maguziano in villa, sans faire explicitement référence à une communauté monastique, mais à un lieu de culte situé dans noyau rural habité59. Toutefois le document mentionne bien

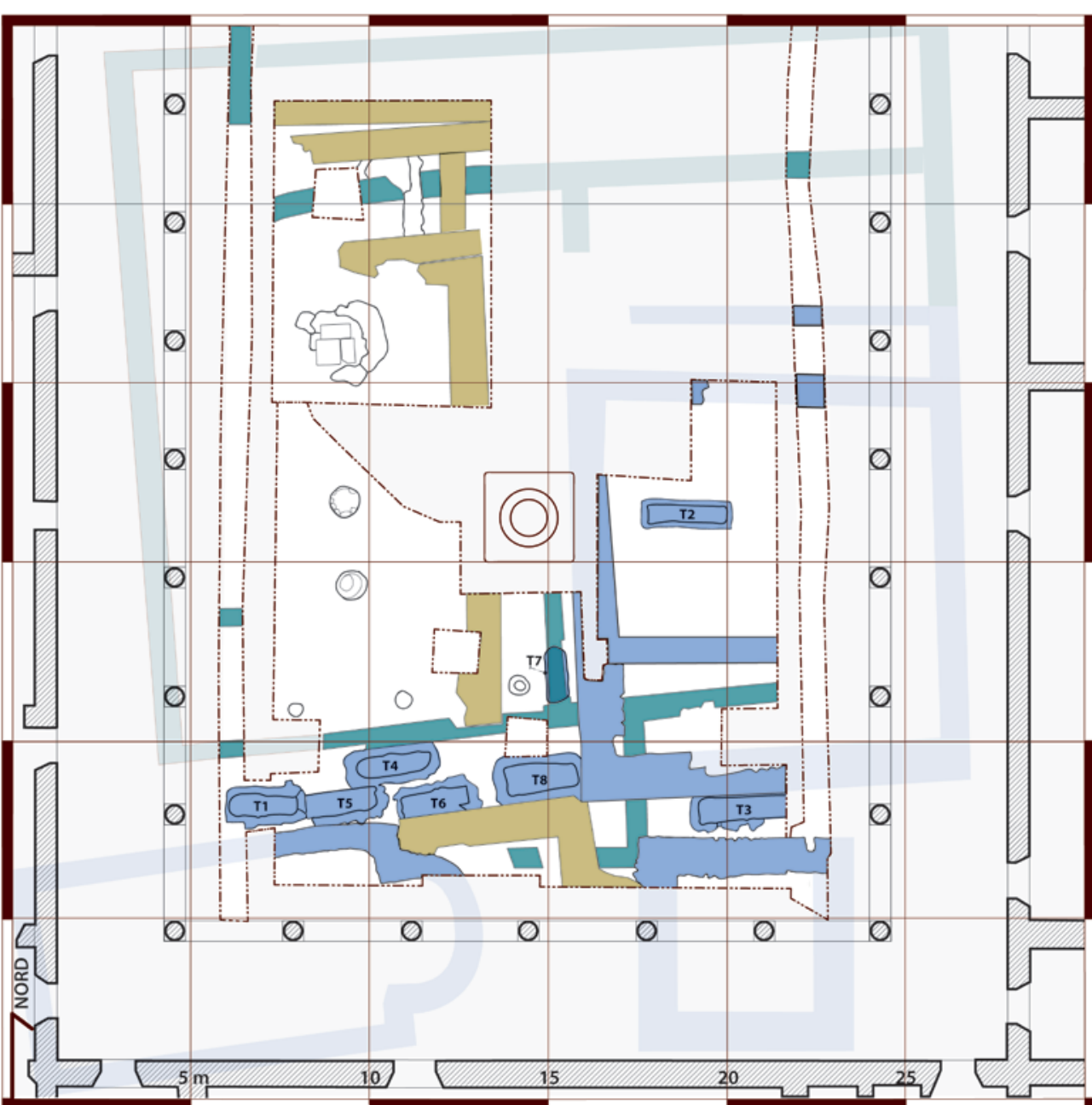
PÉRIODE 3 - VIII ${ }^{\text {S SIECCLE }}$

PÉRIODE 4 - IX-X|e SIÈCLE

PÉRIODE 5

Figure 6. Maguzzano (Lonato-BS), plan des structures avec le détail de l'église et des sépultures funéraires de la seconde phase du haut Moyen Âge des IXe-Xe siècles (d'après A. CHAVARRIA ARNAU, Il monastero..., op. cit. [n. 57], fig. 1, p. 482, modifiée)

un monastère et une église ayant des fonctions paroissiales : non seulement le coenobium, disposant de chapelles et de dîmes, mais aussi une « plebem eiusdem loci cum decimis e capellis suis », ce qui montre implicitement deux entités ecclésiastiques distinctes, chacune gérant ses biens propres ${ }^{60}$.

Il est impossible d'établir un rapport direct entre ce qui a été repéré par les fouilles et le contenu de la source documentaire - ce qui empêche de déterminer à laquelle des deux entités se rapportent les découvertes archéologiques. On est assuré néanmoins qu'un bâtiment d'habitation (II) subdivisé en plusieurs pièces, disposant d'une cour interne et d'un puits est construit au VIII' siècle. La structure se caractérise par des cloisons formées de blocs morainiques irréguliers liés à l'argile, alors que les parements de maçonnerie sont enduits d'un mortier pauvre en chaux ; le sol en argile battue a été recouvert de minces niveaux organiques, contenant des charbons et un foyer ${ }^{61}$ (fig. 6).
Entre le $\mathrm{IX}^{\mathrm{e}}$ et le $\mathrm{XI}^{\mathrm{e}}$ siècle sont édifiés trois nouveaux bâtiments aux fonctions distinctes : le premier (III) est résidentiel, le deuxième (IV) correspond à une église, le troisième $(\mathrm{V})$ à une tour. L'habitation (III), établie pardessus la précédente (II), présente plusieurs phases de construction, une distribution plus compacte des pièces, des maçonneries réalisées à l'aide de pierres soigneusement choisies et ébauchées, liées d'abord à l'argile puis par un mortier grossier de couleur blanchâtre. La fouille de l'une des pièces a mis en évidence des parois enduites à la chaux et peintes en rouge avec des figures faiblement préservées, dont les caractéristiques permettent de supposer qu'il s'agit d'une chapelle funéraire. La surface du sol en mortier présente une découpe rectangulaire destinée à insérer une sépulture maçonnée (sép. 2), orientée Est/Ouest, construite en deux temps. Le côté oriental a été réalisé d'abord à l'aide de pierres ébauchées, puis agrandi vers l'Ouest, par l'ajout

${ }^{57}$ A. CHAVARRÍA ARNAU, Il monastero altomedievale di Maguzzano (Lonato, BS): scavi 2005-2008, in G. VOLPE \& P. FAVIA (éd.), Atti del V Congresso Nazionale di Archeologia Medievale (Foggia, Manfredonia, 30 settembre-3 ottobre 2009), Firenze, 2009, p. 481.

${ }^{58}$ Ibid., p. 481.

59 Ibid., p. 481.

${ }^{60}$ Ibid., p. 481.

${ }^{61}$ Ibid., p. 483-484. 
de trois assises de blocs de tuf équarris et d'un repose-tête en mortier. Utilisée plusieurs fois au fil des siècles, la tombe a livré huit squelettes qui n'étaient plus en connexion anatomique. Construites avec un grand soin, les parois internes sont encore partiellement enduites et peintes en rouge ; le fond est recouvert par trois grandes tegulae $(51 \times 42 \mathrm{~cm})$; la couverture, qui correspond en partie encore par sa disposition à l'une des dernières fermetures effectuées, était faite de plaques de calcaire blanchâtre ${ }^{62}$.

L'avant-dernier bâtiment (IV) correspond à une église mononef à abside semi-circulaire, abritant plusieurs sépultures. L'une d'elles (sép. 3) a été datée du IX ${ }^{\mathrm{e}}$ siècle par la découverte d'une plaque de chancel fragmentaire, analogue à des éléments sculptés de Sirmione, tandis que les autres sépultures ont fourni des fragments d'enduit peint figuré et des tesselles de mosaïques blanches et bleu foncé, que l’on peut mettre en relation avec le décor du sol et des parois de l'église. Le troisième bâtiment $(\mathrm{V})$ devrait correspondre à une tour, dont le prieur Gezone fit refaire le toit à la fin du XII ${ }^{\mathrm{e}}$ siècle, ce qu'indique le texte gravé sur un chapiteau à imposte ${ }^{63}$.

L'histoire du monastère des SS. Ilario e Benedettoà Dogaletto, sur le bord extérieur de la lagune vénitienne, est liée aux politiques territoriales d'un des lignages aristocratiques lagunaires les plus puissants, celui des Partecipazi. Ce n'est pas un hasard si sa fondation en 819 fut planifiée par le doge Agnello Partecipazio, à l'origine des fondements de toutes les structures qui contribuèrent à la puissance de Venise jusqu'au XVIII' siècle $^{64}$.

Un document alto-médiéval indique qu'Agnello et son fils Justinien, affectèrent au prieur Jean, déjà abbé du monastère de S. Servolo, un territoire et une chapelle, connue par le passé sous le seul nom de S. Ilario, qui existait dès la fin du VIII ${ }^{\mathrm{e}}$ siècle. A ce moment, y est attestée la présence d'un noyau de moines bénédictins qui reçoivent l'usage perpétuel de la chapelle S. Ilario, avec tous les droits agricoles, de mouture et de pêche dans plusieurs zones agricoles et lagunaires ${ }^{65}$. En un bref laps de temps les Partecipazi reconnurent au monastère d'autres propriétés foncières qui englobèrent très rapidement d'amples terrains cultivables, des stations lacustres d'accostage et des xenodochia localisés dans les plaines padouane, vénitienne et trévisane ${ }^{66}$.

Le complexe présente la configuration d'une fondation typique du haut Moyen Âge, le rapport à de hautes institutions politiques, la dotation de vastes propriétés agricoles, une semi-indépendance par rapport aux autorités épiscopales locales, et le fait d'être destiné à remplir autant des fonctions proprement religieuses qu'à renforcer et à contrôler économiquement et politiquement d'amples territoires. On a donc là un modèle historique de type occidental adhérant de manière délibéréeà la Règle bénédictine, suivant les prescriptions de Benoît de Aniane - avec donc une composante carolingienne significative - même si la concession d'exemption fiscale du document de fondation utilisait une formule s'inspirant de modèles linguistiques lombards ${ }^{67}$.

\section{SÉPULTURES ET ALIMENTATION}

Les plus anciennes sépultures du monastère de $\mathrm{S}$. Michele alla Verruca remontent à la période initiale d'occupation du site par la communauté, entre la fin du $\mathrm{X}^{\mathrm{e}}$ et le XII ${ }^{\mathrm{e}}$ siècle ; elles n'ont pas laissé d'évidences structurelles considérables $^{68}$. Il s'agit de quatre tombes (sép. 19, 22, 23, 24) entaillant profondément la surface du banc rocheux, qui détermine la disposition des inhumations ainsi que leur développement sur des plans différents ${ }^{69}$. Deux fosses (sép. 19, 24) ont été creusées dans le substrat affleurant, en suivant sa déclivité naturelle, avec deux feuillures prévues pour poser à l'horizontale une couverture de plaques lithiques presque rectangulaires. Quant à l'orientation des inhumés, la position canonique Ouest/Est, crâne à l'Ouest, pieds à l'Est ${ }^{70}$, est attestée dans toutes les sépultures. Les données paléonutritionnelles indiquent un apport en aliments d'origine végétale et en poisson plus consistant que pour la viande et le lait, ce qui semble confirmer que les moines avaient un régime complet et varié, compensant les périodes de jeûne canonique ainsi que les interdictions alimentaires imposées par la Règle ${ }^{71}$ (fig. 7).

L'église (IV) du monastère de Maguzzano, datée de la première moitié du IX $\mathrm{IX}^{\mathrm{e}}$ siècle, contenait des sépultures, mais on en trouvait aussi à l'extérieur du côté nord (sép. $1,4,5,6,7)$. Contemporaines de sa construction, les trois premières (sép. 1, 4, 5) présentent un plan fusiforme, avec des parois en galets alternant avec quelques briques fragmentaires, liés par une couche abondante de mortier de chaux qui couvrait aussi le fond. La première sépulture (sép. 1) comprenait un oreiller réalisé en mortier, tandis que dans un second cas (sép. 4) on avait utilisé une tuile. Ces deux structures ont été identifiées comme des tombes multiples avec un degré d'exhaustivité partiel des inhumés ; seul l'individu reposant sur le fond - vraisemblablement la déposition primaire - conservait une connexion anatomique acceptable. De plus, on a recueilli des objets de parure (fils d'or, boucles) dans le sédiment de remplissage inférieur, alors que le niveau de comblement supérieur ne contenait que des fragments du décor intérieur de l'église (enduit peint, tesselles de mosaïque, fragment de chancel).

\footnotetext{
${ }^{62}$ Ibid., p. 484-485.

${ }^{63}$ Ibid., p. 485-486.

${ }_{4}^{64}$ D. CALAON, M. FERRI \& C. BAGATO, SS. Ilario e Benedetto (IX secolo). Un monastero del nascente dogado veneziano tra terra e laguna, in Atti del V Congresso..., op. cit. (n. 57), p. 498.

${ }^{65}$ Ibid., p. 499.

${ }^{66}$ Ibid., p. 499.

${ }^{67}$ Ibid., p. 503.

${ }^{68}$ F. SBARRA, Il cimitero: cronologia, organizzazione e aspetti della ritualità, in L'aratro e il calamo..., op. cit. (n. 40), p. 217.

${ }^{69}$ Ibid., p. 217-218.

${ }^{70}$ Ibid., p. 232.

${ }^{71}$ F. BERTOLDI \& R. GIACOMELLO, Analisi paleobiologica e paleopatologica degli inumati, in L'aratro e il calamo..., op. cit. (n. 40), p. $272-273$.
} 


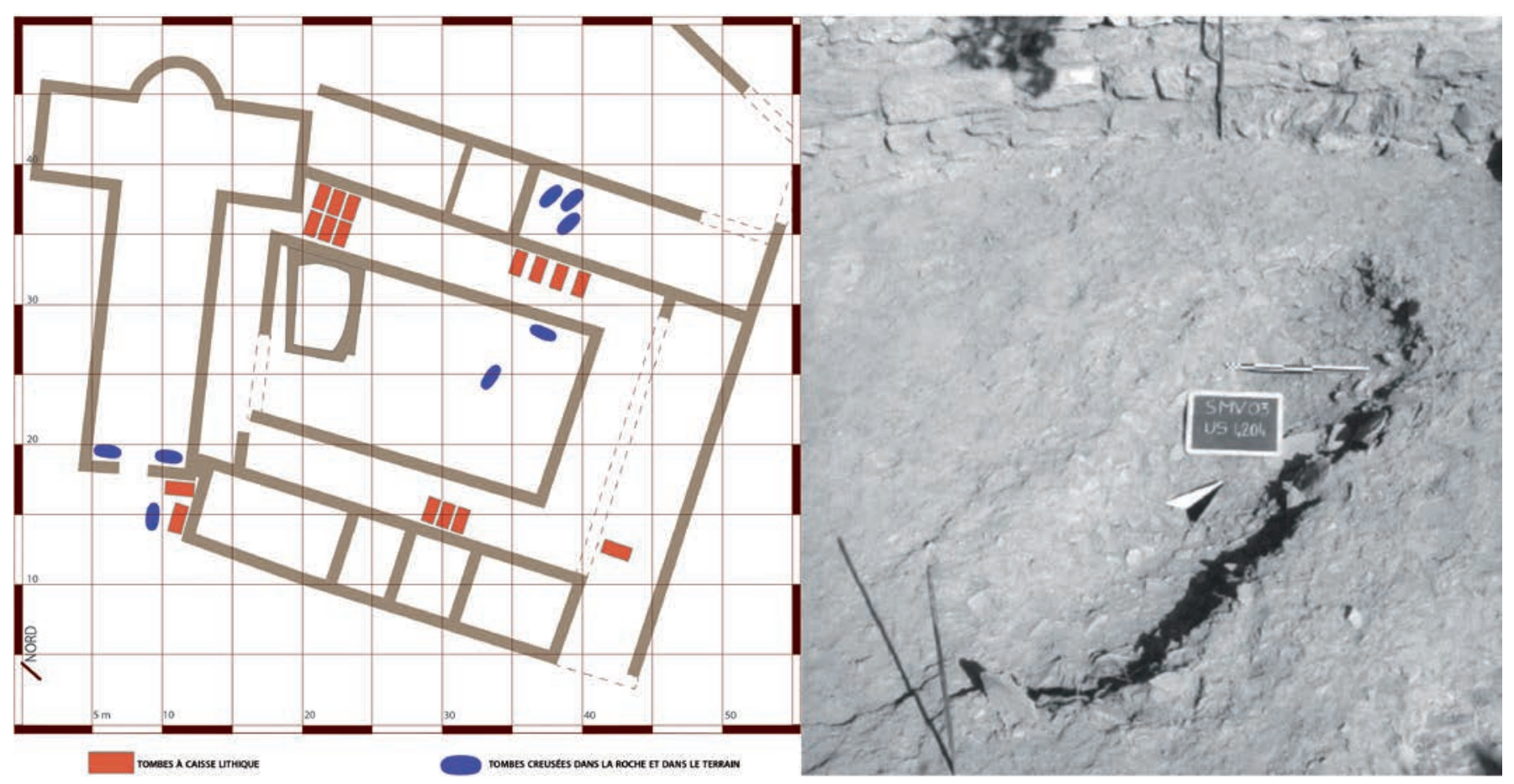

Figure 7. S. Michele alla Verruca (PI), plan général des sépultures fouillées (a), détail de la tombe 23 (b), (d'après F. SBARRA, Il cimitero..., op. cit. [n. 68], fig. 1, p. 218, fig. 18, p. 235)

On en a déduit que les tombes avaient été ouvertes et que les plaques de couverture avaient disparu au moment où l'église fut démolie ${ }^{72}$.

L’une des sépultures examinées présente une épitaphe gravée, avec deux croix, dans le mortier encore frais (sép. 1) : Vberto laicus in pace requiescit. La paléographie et la typologie formelle des croix permettent de proposer une datation entre le $\mathrm{X}^{\mathrm{e}}$ et le $\mathrm{XI}^{\mathrm{e}}$ siècle. Si on accepte la chronologie basse, il est possible de mettre en relation l'inscription et la tombe avec l'Hubert, fils d'Ardoin comte de Parme, présent dans deux actes de 1090 ; dans le premier il offrait une terre agricole à l'église S. Maria de Maguzzano, il était signataire d'un second document rédigé dans la forteresse voisine de Manerba73 (fig. 8).

On situe postérieurement à ces trois premières sépultures, mais avant la construction du bâtiment (VI) au XI siècle, une inhumation de forme rectangulaire (sép. 6), construite avec des pierres ébauchées, disposées en assises horizontales et liées au mortier, avec un repose-tête fait d'une tuile canal à ailettes fragmentaire. Une seule sépulture (sép. 7) avait une orientation Nord/Sud différant de toutes les autres ; elle s'en différenciait aussi par sa fosse simple, où furent déposés, en plusieurs étapes, au moins deux individus, au-dessus desquels avait été étalée une couche de mortier blanchâtre dont la face inférieure conservait l'empreinte d'éléments végétaux (ramilles) et de bois (solives et planchettes), attribuables à une couverture déposée sur le défunt, sur laquelle on avait coulé ensuite du mortier pour sceller la tombe ${ }^{74}$.

Au monastère de $S$. Caprasio on a repéré deux aires funéraires, la première à l'extérieur du chevet, la seconde à l'intérieur de l'église. On y a fouillé un total de 79 sépultures, dont

\footnotetext{
${ }^{72}$ A. CHAVARRÍA ARNAU, Il monastero..., op. cit. (n. 57), p. 484.

73 Ibid., p. 485.

${ }^{74}$ Ibid., p. 485.

${ }^{75}$ E. ARSLAN et alii, Indagini..., op. cit. (n. 36), p. 180.

${ }^{76} \mathrm{Ibid}$., p. 214-216.
}

73 dans la zone extérieure, avec une chronologie comprise entre le haut et le bas Moyen Âge. Y avaient été inhumés des sujets adultes de sexe masculin (32) et féminin (23), des individus immatures (14) pour 24 indéterminés. Seules six sépultures ont été repérées dans l'abside centrale de l'église (sép. $56,57,58 a, 58 b, 59,60)$. Cette partie de l'ensemble religieux étant postérieure aux inhumations, on peut supposer que celles-ci remontent toutes à la phase alto-médiévale, correspondant à la fondation de 884, avec une église et un hôpital gérés et administrés par une communauté monastique qui n'apparaît officiellement que deux siècles plus tard (1077). Des six individus adultes analysés, deux sont assurément de sexe masculin, un n'a pas pu être identifié et les trois autres pourraient être un une femme et deux hommes ${ }^{75}$. Aucun élément n'est susceptible de relier ces sépultures avec l'une des sous-phases du haut Moyen Âge, mais comme on n'a reconnu avec certitude que des individus de sexe masculin, il y a de bonnes probabilités qu'elles puissent être interprétées suivant cette grille de lecture. Les analyses ostéologiques et biologiques ont vérifié de bonnes conditions de santé générale ; deux des défunts présentaient des signes nets d'une usure masticatoire importante ; il n'y avait ni stress physique ni déficience nutritionnelle particulière. On peut prudemment avancer que ces individus appartenaient à une classe sociale aisée, sans carence alimentaire ${ }^{76}$.

Les analyses effectuées sur des squelettes identifiés comme ceux de moines bénédictins du complexe de Westminster, décédés entre le $\mathrm{IX}^{\mathrm{e}}$ et le $\mathrm{XI}^{\mathrm{e}}$ siècle, ont montré que leur régime alimentaire se composait principalement de poisson, d'œufs et de fromage (30\%), de pain (18\%), de vin $(20 \%)$, de plantes potagères complémentaires - tels que oignons, ail, raifort ( $2 \%)$ employés pour assaisonner les 

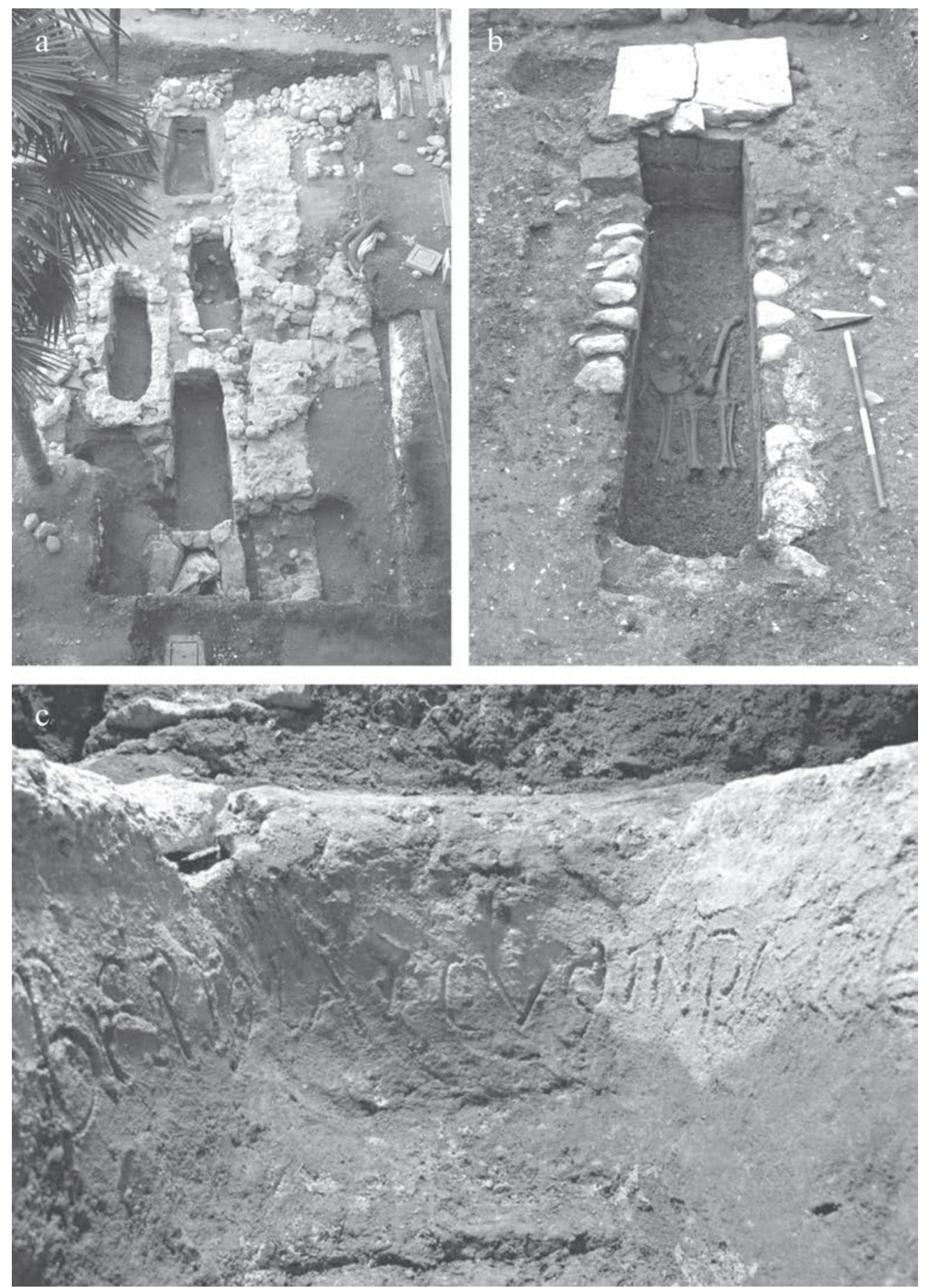

Figure 8. Maguzzano (Lonato-BS) - a. Bâtiment IV (église funéraire) et cimetière correspondant; $b$. Tombe $2 ; c$. Inscription gravée sur la paroi intérieure de la sép. 3 (d'après A. CHAVARRÍA ARNAU, Il monastero..., op. cit. [n. 57], fig. 5-7, p. 484-485)

pulmentaria -, en terminant par des légumes et des fruits $(40 \%)^{77}$. Dans l'échantillon osseux examiné, la présence de quelques isotopes $\left(\mathrm{C}^{13}\right.$ et $\left.\mathrm{N}^{15}\right)$, confirme que poisson, œufs, légumes et fromage étaient les composants principaux d'une alimentation peu diversifiée ${ }^{78}$.
Les analyses paléo-nutritionnelles menées sur ces mêmes squelettes ont montré que le train de vie des moines était nettement supérieurà celui d'autres échantillons de la population rurale contemporaine. Au XI ${ }^{e}$ siècle, chaque moine consommait journellement une quantité légèrement infé-

77 B. HARVEY, Monastic diet, $13^{\text {th }}-18^{\text {th }}$ centuries: problems and perspectives, in S. CAVACIOCCHI (dir.), Alimentazione e nutrizione. Secoli XIII-XVIII, Atti della ventottesima Settimana di Studi dell'Istituto Internazionale di Storia Economica F. Datini (Prato, 22-27 aprile 1996), Prato, 1997, p. 611-641 ; D. GHILCHRIST, B. SLOANE, Requiem: The Medieval Monastic Cemetery in Britain, London, 2005, p. 210 ; G. ARCHETTI, "Mensura victus constituere"..., op. cit. (n. 7), p. 757-797; ID., I monaci a tavola: norme e consuetudini alimentari, in L. Pani Ermini (éd.), Gli spazi della vita comunitaria, Atti del Convegno internazionale (Roma-Subiaco, 8-1o giugno 2015), Spoleto, 2016 (De re monastica, 5), p. 305-327 ; ID., "Parvula poma sumebat". Suggestioni dal mondo monastico, in I. Naso (éd.), Le parole della frutta. Storia, saperi, immagini tra medioevo ed età contemporanea, Torino, 2012 (Centro studi per la storia dell'alimentazione e della cultura materiale "Anna Maria Nada Patrone”), p. 67-89; ID., "Dulcissimas ficus comedere”. Note sparse dalle fonti monastiche, in I. NASO \& C. LITTARDI (éd.), Il fico. L'albero e i suoi frutti tra storia, letteratura, arte, botanica, Atti del convegno internazionale di studio (Sanremo-Bordighera, 22-23 maggio 2015), Imperia 2017, sous presse. ${ }^{78}$ B. HARVEY, Living and Dying in England, 1110-1540: the monastic experience, Oxford, 1993, p. 55-65 ; G. ARCHETTI, "Vas optimo lacte plenum". Latte e formaggio nel mondo monastico, in ID. \& A. BARONIO, La civiltà del latte. Fonti, simboli e prodotti dal Tardoantico al Novecento, Atti dell'incontro nazionale di studio (Brescia, 29-30 maggio 2008), Brescia, 2011 (Storia cultura e società, 3), p. 249-278. 
rieure à $1 \mathrm{~kg}$ de nourriture (poisson, viande ou œufs), environ 4,5 l de bière et une miche de pain - ce type d'alimentation pouvant être difficilement associé à un style de vie ascétique $^{79}$. Les résultats qui découlent de ces analyses montrent qu'en raison du faible apport en fruits et en végétaux frais, le régime des moines pourtant assez diversifié, provoquait une carence de vitamines $\mathrm{A}$ et $\mathrm{D}$, malgré la quantité considérable de poisson et d'œufs régulièrement consommés. Ce déficit alimentaire pourrait avoir été causé par la cuisson des légumes et des plantes potagères, ainsi que par la consommation de poissons séchés et salés, qui diminue d'environ $70 \%$ les précieuses substances vitaminiques ${ }^{80}$. De plus, les moines devaient ressentir davantage des apports protéiques et glucidiques excessifs, liés à la consommation continue de légumes. Un régime alimentaire de ce type contribuait au développement de nombreuses maladies dyscrasiques ainsi que l'incidence considérable d'hyperostose relevée dans beaucoup de sépultures provenant de contextes monastiques datables entre la fin du IX ${ }^{\mathrm{e}}$ et le $\mathrm{XV}^{\mathrm{e}}$ siècle ${ }^{81}$.

Leur espérance de vie, en général très bonne, du moins par rapport aux laïcs contemporains, était probablement due non tant aux habitudes alimentaires qu'au rythme régulier des repas, à celui des heures canoniques qui structuraient la journée de prière et de travail, sans laisser de temps à l'oisiveté, un rythme déterminé par le calcul monastique de la lumière du jour et de l'obscurité de la nuit. La longévité des moines bénédictins et leurs bonnes conditions générales de santé peuvent même impressionner, si l'on considère les alternances perpétuelles entre abstinence, interdiction de consommer certains aliments et régime subséquent peu équilibré de leurs rations journalières : ils ingéraient des glucides farineux et des légumes secs riches en protides dans des quantités qui semblent aujourd'hui insoutenables, même si elles se trouvaient équilibrées par les vitamines et les sels minéraux contenus dans les plats de végétaux crus. Il va cependant sans dire que ces derniers étaient conditionnés par les saisons et les situations climatiques de chaque monastère ${ }^{82}$.

La Règle de saint Benoît prescrit un régime alimentaire très simple mais équilibré du point de vue nutritionnel, en état de satisfaire les besoins énergétiques des moines, même engagés dans des travaux agricoles normalement exclus pour les cénobites des autres ordres. Ce genre d'alimentation sobre et régulier fut considéré comme le plus valable, parce qu'il évoquait et réalisait déjà dans le "monde des vivants" la Jérusalem céleste, où la nécessité de se nourrir aurait disparu $^{83}$. Sur le plan nutritionnel, cette renonciation volontaire n'impliquait pas toujours un refus de la viande, des épices et des repas composés, communs dans la classe moyenne supérieure de la population. On préférait ne pas appliquer de façon univoque le type de choix alimentaire simple et pauvre du monde paysan, fondé principalement sur les produits végétaux, qui aurait été pourtant plus conforme au respect des prescriptions établies par la Règle, tout comme aux idéaux, non écrits, implicitement prévus par l'adhésion à la vie monastique.

La sensation de faim présumée continue et la pénurie de viande dans la nourriture des communautés monastiques - qui n’a jamais été effectivement démontrée pour les premiers siècles du Moyen Âge - étaient compensées par une consommation presque globalisante de légumes et de féculents ${ }^{84}$. Les pratiques d'abstinence, pour lesquelles il est plus correct d'utiliser le mot précis de jeûne imposé - quoique ce ne fût pas une interdiction absolue - sauf à la quadragésime, consistaient en un seul repas journalier, différé à la neuvième heure ou à celle du crépuscule et donc pas à une abstention totale de nourriture ${ }^{85}$.

\section{PRODUIRE, CONSOMMER, GÉRER ET ADMINISTRER}

On a cru longtemps que les monastères du haut Moyen Âge n'étaient que des centres indépendants, de production et de consommation. Cette idée a été démentie il y a des années lorsque l'on a vu que ces communautés monastiques étaient non seulement engagées intensément dans le commerce elles devaient disposer de produits manufacturés ne pouvant être autoproduits - mais qu'en outre elles vendaient le surplus de leur production agricole ou fromagère, certes à bas prix, en se contentant de faible profits. La conception même des complexes monastiques prévoyait des installations spécifiques pour l'élevage, les activités agricoles ; on trouvait ainsi des lieux pour élever des espèces aviaires variées et de nombreux animaux (chèvres, moutons, chevaux, bovins), fondamentaux pour les activités de production du monastère, mais aussi des cuves pour le poisson, des porcheries dont la présence ne se justifie que par la production et le traitement de viande (salée ou fumée), de saucisses et de dérivés carnés ${ }^{86}$.

Dans la zone-témoin que constitue le territoire ligure en termes de diffusion des cellules de Bobbio, il n'est guère surprenant que dans le contexte urbain important de Genua, ancien municipe romain, le plus ancien monastère soit justement celui d'une communauté de moines colombaniens qui contrôlait l'église S. Pietro de Porta entre le milieu du IX $^{\mathrm{e}}$ et la fin du $\mathrm{X}^{\mathrm{e}}$ siècle $(862-972)^{87}$.

Il ne faut pas imaginer les annexes de Bobbio dans ce secteur territorial de façon casuelle et fortuite, mais plutôt comme le résultat de choix stratégiques planifiés : la présence monastique garantissait une organisation économique

79 E. CIRELLI, La dieta dei monaci..., op. cit. (n. 18), p. 230.

8o Ibid., p. 231.

${ }^{81}$ L. MOULIN, La vita quotidiana secondo San Benedetto, Paris, 1982, p. 223 ; A. D’HAENENS, La quotidienneté monastique au Moyen Âge, Paris, 1984, p. 31-42 ; G. ZIMMERMANN, Ordensleben und Lebensstandard. Die “Cura Corporis” in dem Ordensvorschriften des abendländischen Hochmittelalters, Münster, 1973 ; G. ARCHETTI, “Nihil operi Dei praeponatur”. Il tempo dei monaci nel Medioevo, in ID. \& A. BARONIO, “Tempus mundi umbra aevi”. Tempo e cultura del tempo tra Medioevo e età moderna, Atti dell'incontro nazionale di studio (Brescia, 29-30 marzo 2007), Brescia, 2008 (Storia, cultura e società, 1), p. 51-80.

${ }^{82}$ A. SÉGUY, Une sociologie des sociétés imaginées : monachisme et utopie, Paris, 1981, p. 328-355.

${ }_{3}^{3}$ M. MONTANARI, L'alimentazione contadina nell'alto medioevo, Napoli, 1976, p. 28-29; mais surtout G. ARCHETTI, "Mensura victus constituere”... , op. cit. (n. 7), p. 757-797.

${ }^{84}$ A. SCARNERA, Il digiuno cristiano dalle origini al IV secolo, Roma, 1990, p. 74-82.

${ }^{8}$ E. CIRELLI, La dieta dei monaci..., op. cit. (n. 18), p. 231.

${ }^{86}$ Ibid., p. 230 ; E. DESTEFANIS, Il monastero di Bobbio in età altomedievale, Firenze, 2002 (Ricerche di archeologia altomedievale e medievale, 27), p. 50-51.

${ }_{7}^{8}$ V. POLONIO, Il monachesimo femminile in Liguria dalle origini al XII secolo, in G. ZARRI (dir.), Il monachesimo femminile in Italia dall'alto medioevo al secolo XVII a confronto l'oggi, Negarine di S. Pietro in Cariano, 1997, p. 90 ; E. TORRE, Da Genua a Ianua, in P. MELLI (dir.), Genova. Dalle origini all'anno Mille. Archeologia e storia, Genova, 2014, p. 240. 


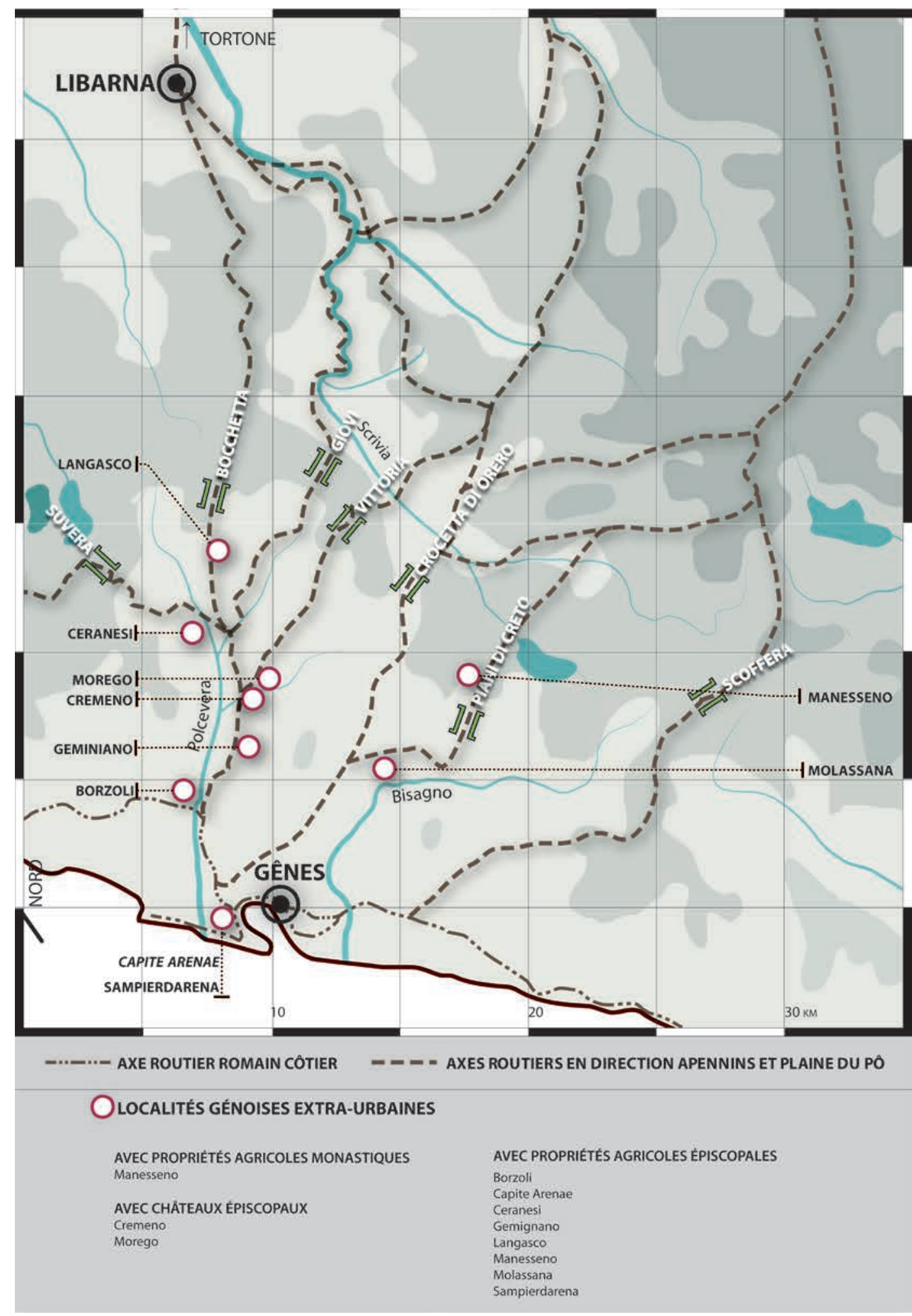

Figure 9. Tableau global des possessions monastiques, épiscopales et féodales dans les zones périphériques et rurales de Gènes (C) Rossana Managlia)

efficace, alors que la qualité du climat tempéré permettait d'obtenir des denrées primaires (huile, vin, sel, châtaignes), secondaires (figues, cédrats) et un produit complémentaire tel que la poix, essentielle à la commercialisation et à la conservation, au moins partielle, de certains aliments spécifiques $^{88}$.

Le patrimoine du monastère bénédictin de $\mathrm{S}$. Siro fut défini dès sa naissance, à la suite d'importantes donations, comme celles de l'évêque Théodulf en 952 et d'Adélaïde de Bourgogne en 989 ou encore celles de particuliers, avec des biens offerts à Beleno, sur les hauteurs de Manesseno dans le Val Polcevera en l'an Mil ${ }^{89}$. L'embouchure du fleuve Polcevera offrait un point de passage aisé vers le bassin contigu du Bisagno. Ce torrent côtier qui traverse la partie occidentale de Gênes, ouvre à son tour d'autres possibilités de liaison en Ligurie orientale et même au-delà des Apennins, vers

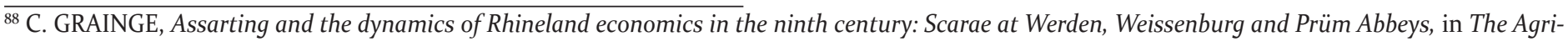
cultural History Review, 54.1, 2006, p. 1-23.

${ }^{89}$ S. ORIGONE, Il patrimonio immobiliare del monastero di S. Siro di Genova (secoli X-XIII), in Studi Genuensi, 10, Genova, 1973-1974, p. 3-14. 
la Plaine du Pô. Rappelons enfin la dotation de l'évêque Jean II dans un acte par lequel il nommait le premier abbé en 1007 , en la personne du moine Pierre « et pro delictorum nostrorum nostrorumque precedentium ac successorum venia, quondam monachum, nomine Petrum, cum omnibus monachis ${ }^{90}$, en accordant la transmission de plusieurs dîmes et de biens immobiliers situés à Gênes et dans son voisinage immédiat, contre la rémunération d'un cens annuel de 6 muids plenos potione et le même nombre de cierges ${ }^{91}$.

Ce document marque la première pierre d'un important projet politique bien planifié. Le caractère problématique des relations entre la vieille et la nouvelle cathédrale, entre les pouvoirs religieux, comtal et vicomtal, entre les priorités du noyau citadin et celles du développement des implantations suburbaines dans le cadre de la civitas, y trouvent tous un dénominateur commun avec la présence génoise le long des parcours routiers conduisant vers la Plaine du Pô et les deux littoraux, du Levant et dans une mesure nettement inférieure du Ponant ${ }^{92}$.

Jean II confirma au monastère le contrôle d'un vignoble prolifique, à proximité de l'église $\mathrm{S}$. Siro, que son prédécesseur Théodulf avait récupéré après son usurpation par le prêtre Sylvestre et remis à S. Siro en 952. L'évêque confirma la perception de dîmes " ad suprascriptam ecclesiam pertinentes ", dont on ignore si elles concernaient tout le faubourg, pourtant dévolu au collège sacerdotal par Théodulf en 952, ou une partie de celui-ci. Jean II enrichit le patrimoine épiscopal de propriétés foncières situées près de S. Michele in Capite Arenae, qu'on reconnaît dans le secteur nord de la partie la plus ancienne de Gênes, à proximité de la Gare Maritime93. En réalité ces biens sont minces par rapport au transfert de terres plus substantielles dans le Val Polcevera : Borzoli, Ceranesi, Beleno et Langasco ${ }^{94}$. Ce ne sont là que les premières étapes d'un processus d'agrandissement, qui se poursuivra dans la première moitié du $\mathrm{XI}^{e}$ siècle, par de nouvelles acquisitions dans le territoire génois au sens large, toujours dans le Val Polcevera à Gemignano, mais aussi à Sampierdarena, à Cogoleto et dans le Bas Piémont ${ }^{95}$ (fig. 9).

La position de prestige que conférait la fonction de première cathédrale de Gênes, les liens étroits avec les vicomtes ${ }^{96}$, qui avaient à S. Siro leurs caveaux de famille, les rapports politiques qui liaient les abbés aux événements du $\mathrm{XI}^{\mathrm{e}}$ siècle, contribuèrent à un développement patrimonial de grande envergure pour le monastère ${ }^{97}$ - soulignant la profondeur des interconnexions avec le monde politique féodal ${ }^{98}$.

Les sources textuelles contribuent de manière significative à mettre en évidence les bâtiments religieux du haut Moyen Âge, en incluant des églises et fondations monastiques, qui ont disparu par la suite - et donc à fournir un tableau plus complet et exhaustif de la période - mais aussi des interrelations entre une ville en expansion continue et son faubourg immédiat. À tout ceci se greffent les intérêts de la Curie archiépiscopale qui dictèrent le rythme de la politique et des acquisitions territoriales, notamment celles du monastère de S. Siro, dans un schéma de mise en œuvre qui semblait positivement devancer les événements. On peut ainsi affirmer qu'au $\mathrm{X}^{\mathrm{e}}$ siècle les intentions sont déjà clairement définies pour les vallées de la Polcevera et du Bisagno, et qu'elles suivent une direction univoque. Les différentes institutions monastiques contrôlaient les nombreuses propriétés distribuées sur ce territoire, selon des répartitions et des superficies différentes, les aires les plus significatives étant localisées dans les parties médiane et supérieure des deux vallées 99 .

Le paysage agricole, appelé dans les documents vineato et arborato, s'organisait en fundi, sur lesquels les monastères citadins exerçaient la fiscalité des dîmes et dont la possession était confirmée par des actes d'achat/vente et de location. Productif et irrigable, il comprenait des moulins et des aqueducs. Certains documents antérieurs au XI ${ }^{\mathrm{e}}$ siècle mentionnent des propriétés foncières de la Curie génoise, mais aussi la production de plantes potagères, le traitement des viandes de porc et de mouton de la curtis de Molassana, qui ravitaillait le réfectoire épiscopal. Il est plausible d'envisager des liaisons et des parcours routiers à longue et moyenne distance ${ }^{100}$.

La présence d'une structure épiscopale fortifiéeà Cremeno dans le Val Polcevera constitue un élément important et indicatif, qui augmente la valeur stratégique de cette vallée fluviale débouchant sur la mer à Gênes. De là une voie de communication directe favorable permettait de véhiculer hommes et marchandises vers le Piémont et la Lombardie ${ }^{101}$.

\footnotetext{
${ }_{90}^{\circ}$ M. CALLERI, Le carte del monastero di S. Siro di Genova (952-1224), I, Fonti per la Storia della Liguria, V, Società Ligure di Storia Patria, Genova, 1997, document 15, p. 24-27.

${ }^{91}$ M. CALLERI, Le carte del monastero, op. cit., document 15, (n. 90), p. 24-27.

$9^{2} \mathrm{P}$. DE VINGO, Il potere della fede. Gestione del territorio e delle sue risorse, produttività agricola dei monasteri urbani genovesi tra X e XI secolo, à paraître.

${ }^{93}$ M. CALLERI, Le carte del monastero di S. Siro, op. cit., document 15, (n. 9o), p. 24-27.

${ }_{94}$ R. RINALDI, L'incolto in città, in B. ANDREOLLI \& M. MONTANARI (éd.), Note sulle vicende del paesaggio urbano tra alto Medioevo ed età comunale, Bologna, 1988, p. 250-262.

${ }_{95}$ P. DE VINGO, Il potere della fede..., op. cit. (n. 92).

${ }_{96}^{6}$ Le marquis Obert, fils de feu Obert en 1014, le comte Gaidald, fils de feu Ingon en 1017, le vicomte Gandolf, fils de feu Guillaume en 1030 et les vicomtes des trois branches de Carmadino, d'Isola et de Manesseno en 1052.

${ }_{97}$ V. POLONIO, Da Provincia a Signora del mare (secoli VI-XIII), in D. PUNCUH (dir.), Storia di Genova. Mediterraneo, Europa, Atlantico, Genova, 2003, p. 127-128.

${ }_{98}^{8}$ P. DE VINGO, Il potere della fede..., op. cit. (n. 92).

${ }^{99}$ ID., Note su alcune fondazioni religiose a Genova e nel territorio suburbano tra X e XI secolo, in R. SALVARANI, G. ANDENNA \& G.P. BROGIOLO (éd.), Alle origini del romanico. Monasteri, edifici religiosi, committenza tra storia e archeologia (Italia settentrionale, secoli IX-X), Atti delle III Giornate di Studi Medievali (Castiglione delle Stiviere, 25-27 settembre 2002), Studi documenti 3, Centro Studi sulla Storia degli Insediamenti Monastici (CESIMB), Brescia, 2005 , p. 213 -236. ${ }^{100}$ P. GUGLIELMOTTI, Ricerche sull'organizzazione del territorio nella Liguria medievale, Firenze, 2005, p. 15-47 ; E. TORRE, « Da Genua.... », op. cit. (n. 87), p. 241-242.

${ }^{101} \mathrm{~F}$. BENENTE, Incastellamento e poteri locali in Liguria. Il Genovesato e l'area del Tigullio, in ID. (éd.), L'incastellamento in Liguria (X-XII secolo). Bilancio di un tema storiografico (Rapallo, 26 aprile 1997), Bordighera, 2000 (Istituto Internazionale di Studi Liguri. Atti dei Convegni, IV), p. 48 ; G. ARCHETTI, "Vineam noviter pastinare". Note storiche sulla vite e sul vino nella Liguria medievale, in A. CARASSALE \& L. LO BASSO, "In terra vineata". La vite e il vino in Liguria e nelle Alpi Marittime dal Medioevo ai nostri giorni, Atti del convegno di studi in memoria di Giovanni Rebora (Taggia, 6-8 maggio 2012), Ventimiglia, 2014, p. 13-35.
} 

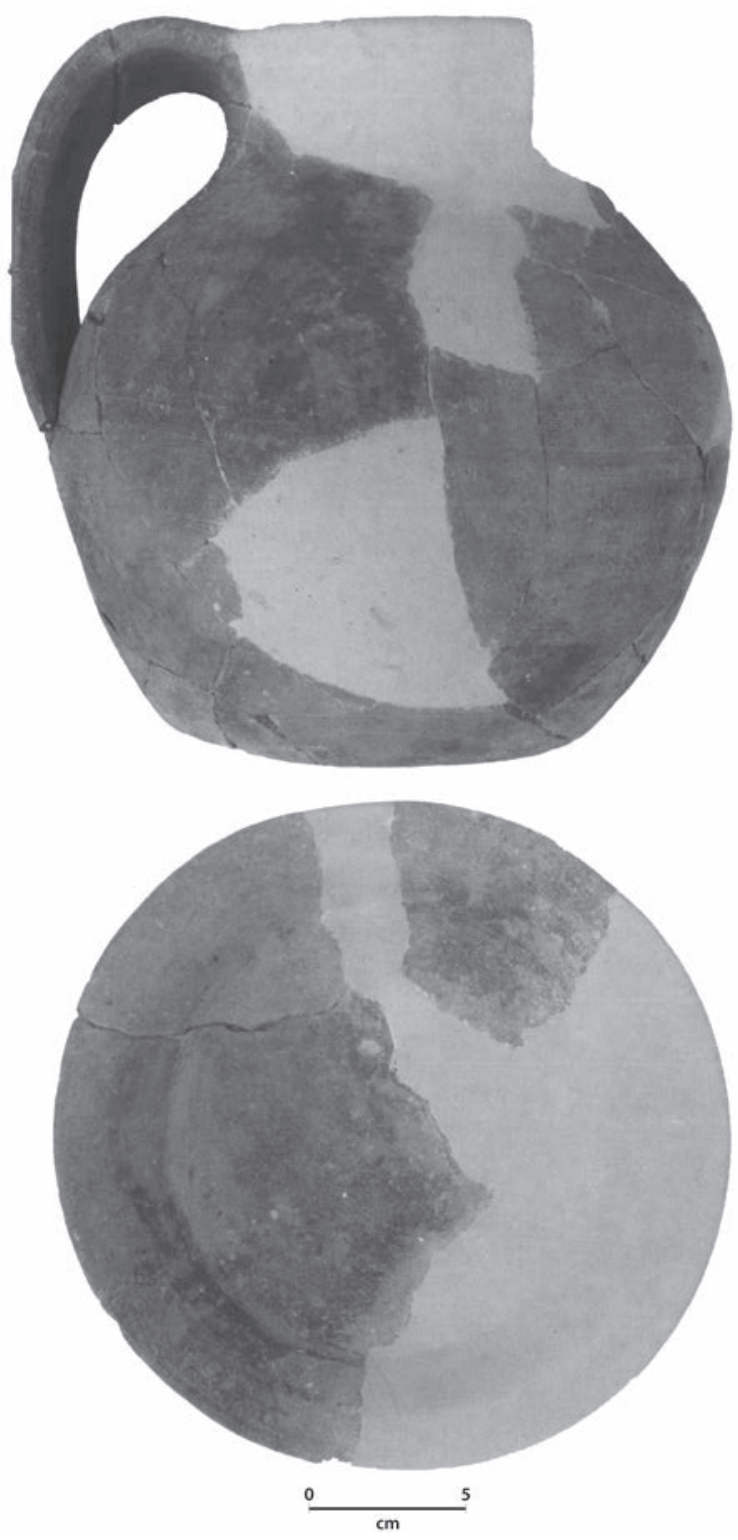

Figure 10. S. Michele alla Verruca (PI), vaisselle de la première phase de l'établissement-a. Bocal; b. Plat à pain (testello) en céramique brute (d'après A. ALBERTI, S. BARTALI \& S. BOSCOLO, Le ceramiche..., op. cit. [n. 106], fig. 3-4, p. 278)

Ce système routier, demeuré suffisamment efficace et praticable après le VI ${ }^{\mathrm{e}}$ siècle, fut en mesure de raccorder aussi le territoire urbain avec son faubourg immédiat, en sus du dense réseau secondaire qui traversait et reliait les campagnes ${ }^{102}$.

On ne dispose pas de données quantitativement significatives pour réfléchir aux caractéristiques des terrains achetés. Les éléments qui renseignent les productions ou les types de cultures pratiquées s'avèrent intéressants, mais on ignore par exemple les mesures agricoles des pecie individuelles de terre. Il est par conséquent impossible d'évaluer si l'on est en présence d'une parcellisation extrême du terrain et si les acquisitions éventuelles répondent, à travers une politique attentive et prudente, à la formation d'extensions plus homogènes et contiguës. Deux documents, de 993 et de 1079, indiquent que « quod est per censura in giru et circuitu perticas nonaginta et tres a pertica de pede duodecim $[\ldots] »$ dans le premier, et que «[...] et est predicta pecia de terra per censura iusta tabulas quadraginta et due a pedes » dans le second ${ }^{103}$.

En 958 la reconnaissance du droit coutumier, la limitation des pouvoirs exécutifs des officiers publics et l'exemption $\mathrm{du}$ mansionaticum par Bérenger II et Adalbert en faveur de Gênes - en raison de la capacité entrepreneuriale des vicomtes à réinvestir le surplus des rentes agricoles dans la construction de navires, de maisons et d'entrepôts - sanctionnèrent une autonomie citadine précoce, porteuse de ce que sera le développement communal. De la même façon, l'Église dont les propriétés du Val Polcevera touchaient celles des Carmadino et des Isola, investit dans la fortification du château près de l'ancien oppidum préromain, nouveau pôle diffuseur de la civitas de l'an Mil. On entrevoit ainsi une société très articulée, dotée de capacités matérielles et d'entreprise, formée d'aristocrates, de marchands, de clercs, de juges et de juristes ou bien de représentants d'une classe fonctionnelle à la vie urbaine active, organisée et productive ${ }^{104}$.

\section{CONSOMMATION ALIMENTAIRE ET VAISSELLE DOMESTIQUE DANS LES MONASTÈRES ENTRE LE VII ${ }^{\mathrm{e}}$ ET LE XI ${ }^{\mathrm{e}}$ SIÈCLE}

Du point de vue de la céramique de préparation, cuisson et conservation des aliments, la typologie des produits manufacturés dans les centres monastiques examinés ne se différencie pas beaucoup de celle des autres contextes de consommation médiévaux du centre-nord de la péninsule italienne. À S. Caprasio, on utilisait au haut Moyen Âge des conteneurs non colorés, des oules brutes à parois fines et fond plat, à stries de tournage parfois marquées et bords légèrement repliés vers l'extérieur, des "testelli" (plats à pain) et des récipients en pierre ollaire. Parmi les nombreux fragments de verre, on a même un goulot de bouteille; dans les cas où l'on peut déduire la forme, il s'agit en général de calices à bord légèrement épaissi. Deux tessons vitreux présentent un décor à bandes et à encoches blanches en relief ou alternant avec de fines lignes blanches et vert-bleu ${ }^{105}$.

La culture matérielle relative à la phase monastique initiale du site de $\mathrm{S}$. Michele alla Verruca est très pauvre. Elle se limite à trois exemplaires probables de brocs en céramique épurée, à des oules et brocs à pâte grossière, un plat à pain (testello) et une casserole. Par contre, les contextes concernant les phases de fréquentation du monastère comprises entre la fin du X $\mathrm{X}^{\mathrm{e}}$ et le début du XII ${ }^{\mathrm{e}}$ siècle comprennent beaucoup plus de fragments de céramique épurée ou commune et de pierre ollaire, qui ramènent à des exemplaires de brocs à pâte fine, avec dans un cas de légères bandes peintes en brun sur la surface extérieure. Les conteneurs à pâte grossière correspondent à de petits brocs ou bocaux, dans certains cas utilisés pour réchauffer des liquides par réverbération du feu, alors que d'autres renvoient à des oules, des "testelli" et des marmites ${ }^{106}$ (fig. 10).

${ }^{102}$ P. DE VINGO, A. FRONDONI, Fonti scritte e cultura materiale del territorio fra tardoantico ed altomedioevo in Valpolcevera (Genova): problemi aperti e prospettive, in R. FIORILLO \& P. PEDUTO (dir.), Atti del III Congresso Nazionale di Archeologia Medievale (Salerno, 2-5 ottobre 2003 ), Firenze, 2003, p. 32. ${ }^{103}$ P. DE VINGO, Il potere della fede..., op. cit. (n. 92).

${ }^{104}$ Ibid.

${ }^{105}$ E. ARSLAN et alii, Indagini..., op. cit. (n. 36), p. 180-182.

${ }^{106}$ A. ALBERTI, S. BARTALI \& S. BOSCOLO, Le ceramiche dei monaci e dei soldati, in L'aratro e il calamo..., op. cit. (n. 40), p. 276-279. 
Les fouilles de S. Fruttuoso de Capodimonte ont livré une quantité considérable de céramiques destinées à la préparation de la nourriture et au service de la table, notamment pour les $\mathrm{X}^{\mathrm{e}}-\mathrm{XI}^{\mathrm{e}}$ siècles. Parmi les céramiques culinaires se distinguent des casseroles et marmites glaçurées, même si la cuisine du monastère cuisait plutôt les aliments dans des récipients cylindriques en pierre ollaire. La présence de fragments d'amphores médiévales témoigne du transport, du stockage et de la conservation de liquides, vraisemblablement du vin, vu son importance comme complément alimentaire et dans la célébration eucharistique. À table on utilisait de grands récipients collectifs (cuvettes), des bocaux pour le débit des liquides (eau et vin), des bols et des gobelets en verre. D'autres formes ouvertes plus petites, telles que des jattes et des bols, servaient aux sauces et aux épices. Les assiettes étaient utilisées individuellement par chaque convive ${ }^{107}$.

Les reconnaissances effectuées dans les zones occupées par le monastère des SS. Ilario e Benedetto à partir de sa fondation au IX ${ }^{\mathrm{e}}$ siècle, ont permis de recueillir un nombre majeur de fragments de conteneurs de transport, suivis par la vaisselle en pierre ollaire, les céramiques du type "S. Alberto" et la céramique commune épurée ${ }^{108}$. Tandis qu’à $S$. Fruttuoso de Capodimonte le nombre minimum d'individus (NMI) des amphores rend plausible leur utilisation pour le stockage de liquides, de vin en particulier, la situation est exactement contraire dans le cénobe lagunaire, dans le sens où l'on ne pense pas seulement à une forme de ravitaillement pour la communauté monastique, mais plutôt à la présence d'entrepôts et de magasins, où les amphores étaient rangées. Dans ce cas le NMI est compatible avec des activités de commercialisation de moyenne et longue distance, même si la typologie précise des produits véhiculés reste inconnue.

\section{TEMPS DE POUVOIR, TEMPS DU COMMERCE ET DE L'ÉCHANGE}

Le monachisme bénédictin apparait comme un genre idéal de vie proposé aux laïcs : le moine nétait pas un clericus et n'occupait aucune position précise dans la structure hiérarchique du clergé. Le seul personnage auquel était reconnue une fonction religieuse était l'abbé, qui était généralement prêtre ${ }^{109}$. Les monastères étaient des centres de vie spirituelle et d'éloignement de la matérialité terrestre, du moins dans leurs principes constitutifs, et fréquemment aussi des lieux de sépultures pour les communautés civiles limitrophes. Ils fonctionnaient aussi comme centres de pouvoir fondamentaux pour le développement des appareils économiques territoriaux et pour ceux des aristocraties urbaines. Par conséquent les monastères, où les moines représentaient les familles médiévales européennes les plus importantes, étaient des lieux importants pour la gestion des biens de la part des élites. Malgré le style de vie qu'imposait le choix monastique régi par la Règle bénédictine, les com- munautés monastiques du haut Moyen Âge constituaient une référence incontournable pour les classes sociales aristocratiques et les domaines élitaires de condition élevée, même si l'on n'explique que partiellement quelles étaient les formes de représentation adoptées.

Un exemple concret de ce "temps du pouvoir" est offert par la chapelle alto-médiévale du monastère des SS. Ilario e Benedetto, qui bénéficia de privilèges d'immunité importants. Dès sa fondation elle fut indépendante de l'évêché d'Olivolo et du patriarcat de Grado. Le seul pouvoir qui s'exerçait sur elle était celui de la famille des Partecipazi, montrant de façon significative son caractère de "chapelle privée", de la famille du doge, et tout à la fois d'"espace public" lié aux institutions vénitiennes les plus importantes. Nombreuses sont les similitudes entre le lignage des Partecipazi et les élites familiales présentes sur la terre ferme entre le $\mathrm{VIII}^{\mathrm{e}}$ et le IX ${ }^{\mathrm{e}}$ siècle. On relève une volonté précise d'affirmation de leur condition sociale, corroborée et renforcée par la fondation de maints bâtiments religieux privés. La fondation de cet oratoire privé dans un domaine aussi restreint que celui de la lagune peut donc être évaluée comme si elle avait eu lieu sur la terre ferme, mais avec une signification encore plus marquée, en considérant en outre qu'à la fin du VIII ${ }^{e}$ siècle le pouvoir des Partecipazi était encore en cours de formation et de consolidation ${ }^{10}$. Il en découle clairement que cette famille a su incarner, par des choix adéquats et réfléchis, une aptitude précise à agir en tant que feudataire au sein du Royaume d'Italie naissant. Elle le fit en acquérant des terres et des lagunes et en les faisant gérer par une institution monastique assurément active dans les échanges dès ses origines, et qui se transforma très rapidement en un port lagunaire-fluvial, devenant un avant-poste de contrôle et de gestion des trafics commerciaux au même titre que les accostages de Gambarate et Oriago, situés sur la Brenta comme S. Ilario ${ }^{\mathrm{II}}$.

Parmi les artefacts issus des fouilles des monatères examinés ici, certains éléments se distinguent par rapport aux communautés contemporaines. Il s'agit de céramiques islamiques, que l'on retrouve presque exclusivement dans les contextes monastiques, urbains ou sur des sites commerciaux dynamiques. Ces produits manufacturés sont très rares et pour cette raison ils constituent des marqueurs précieux, surtout si l'on considère que leur circulation entre le IX ${ }^{\mathrm{e}}$ et le $\mathrm{X}^{\mathrm{e}}$ siècle demeure méconnue même dans les aires situées à proximité de leurs lieux de production ${ }^{112}$. On suppose une provenance tunisienne pour une tasse à une anse, glaçurée et peinte, retrouvée au monastère de $\mathrm{S}$. Caprasions. Des vaisselles islamiques glaçurées, identifiables aux luxueuses productions à "lustre métallique", sont documentées aussi bien aux SS. Ilario e Benedetto quà S. Michele alla Verruca ${ }^{114}$.

Ces productions ont été interprétées comme des dons, des échanges, entre des élites de haut niveau social et institutionnel, réaffirmant le rôle de centre du pouvoir

\footnotetext{
${ }^{107}$ A. GARDINI, Gli scavi...., op. cit. (n. 54), p. 26-29.

${ }^{108}$ D. CALAON, M. FERRI \& C. BAGATO, SS. Ilario e Benedetto..., op. cit. (n. 64), p. 500.

${ }^{109}$ G. PENCO, Il monachesimo..., op. cit. (n. 9), p. 74-75.

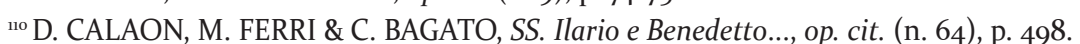

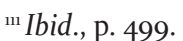

${ }^{122}$ P. CRESSIER \& E. FENTRESS (dir.), La céramique maghrébine du Haut Moyen Âge (VIIIe-Xe siècle). "Urbes Extinctae". Archaeologies of Abandoned Classical Town, Farnham, 2012, p. 45-75.

${ }^{113}$ E. GIANNICHEDDA, R. LANZA \& O. RATTI, Indagini nella canonica e nel chiostro di San Caprasio ad Aulla (MS), in Archeologia medievale, 38, 2011 p. 307. ${ }^{114}$ D. CALAON, M. FERRI \& C. BAGATO, SS. Ilario e Benedetto..., op. cit. (n. 64), p. 498-504; A. ALBERTI, S. BARTALI \& S. BOSCOLO, Le ceramiche..., op. cit. (n. 106), p. 279.
} 


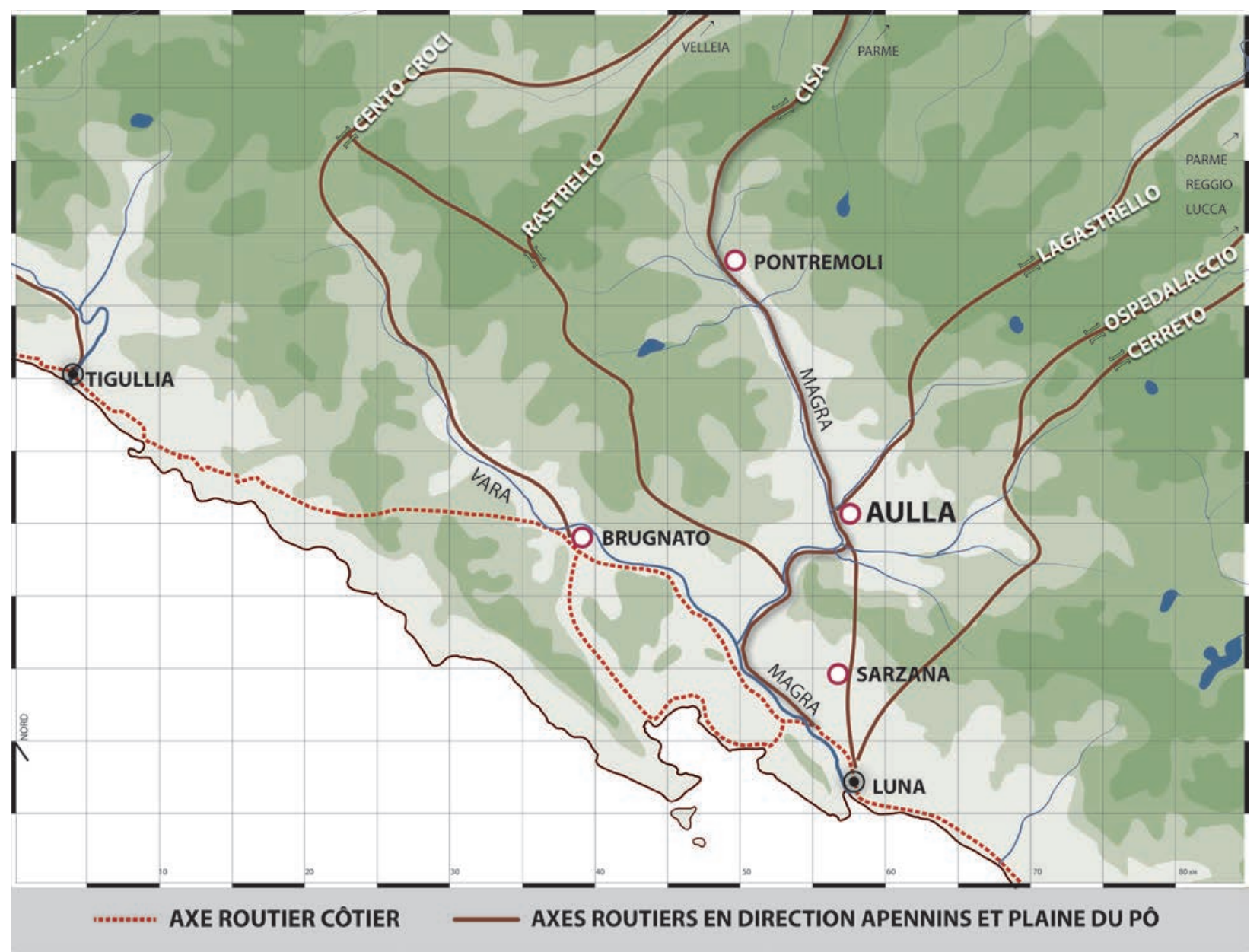

Figure 11. Reconstruction du réseau routier alto-médiéval de la Lunigiane (C Rossana Managlia)

exercé alors par les monastères ${ }^{115}$. En plus des découvertes de luxe déjà évoquées, le matériel archéologique montre que même pour les produits manufacturés d'usage domestique, les communautés monastiques avaient recours à des productions locales et à des échanges commerciaux avec le monde extérieur. En témoignent la vaisselle en céramique fine et les récipients en pierre ollaire, qui apparaissent dans tous les contextes examinés, des plus proches du secteur alpin jusqu'aux plus éloignés, dans l'arrière-pays du littoral Adriatique ${ }^{116}$. La présence importante mais non exclusive d'amphores globulaires datées des VIII ${ }^{\mathrm{e}}-\mathrm{XI}^{\mathrm{e}}$ siècles, souligne l'ouverture des centres monastiques à des transactions commerciales de longue distance. Ce type de conteneur de transport est attesté au monastère des SS. Ilario e Benedetto, proche de la zone majeure de diffusion de ces amphores, principalement liées à la circulation des denrées le long du cours du Pô et dans son delta ${ }^{117}$.

Ce matériel ainsi que la position géographique du cénobe bénédictin définissent son rôle actif dans les échanges à courte et à moyenne distance, vers l'intérieur du continent comme vers les ports maritimes. Cette caractéristique com- merciale des monastères de la péninsule italienne n'est pas une nouveauté dans le panorama du haut Moyen Âge. Ces institutions dépendant souvent des stratégies politiques des pouvoirs centraux, géraient et contrôlaient économiquement des fortunes foncières étendues, souvent éloignées, figurant ainsi parmi les promoteurs des échanges commerciaux de longue et moyenne portée. Malgré tout, faute de données archéologiques suffisantes, il demeure difficile et prématuré d'essayer de quantifier la consistance des échanges et, en dernière analyse, la capacité commerciale du monastère ou même son rôle, qui devait être proportionnel à sa capacité de collecte et de redistribution des marchandises, au vu de sa position médiane en regard des parcours lagunaires ${ }^{118}$.

Permettez-nous encore quelques considérations sur les contextes où l'on a retrouvé des quantités significatives de monnaies ou de restes fauniques. Les investigations menées à $\mathrm{S}$. Michele alla Verruca ont livré peu de restes ostéologiques d'animaux et en l'espèce, dans les limites chronologiques que nous nous sommes imposées $\left(\mathrm{VII}^{\mathrm{e}}-\mathrm{XI}{ }^{\mathrm{e}}\right.$ siècle), un unique fragment de diaphyse de fémur de porc n'autorise aucune évaluation alimentaire. Cependant, on

\footnotetext{
${ }^{15}$ R. HODGES, Ripensando San Vincenzo al Volturno, in Archeologia medievale, 37, 2010, p. 505-506.

${ }^{16}$ D. CALAON, M. FERRI \& C. BAGATO, SS. Ilario e Benedetto..., op. cit. (n. 64), p. 503 ; E. CIRELLI, Anfore globulare a Classe nell'alto medioevo, in Atti del V Congresso..., op. cit. (n. 57), p. 563-568 ; S. GELICHI, C. NEGRELLI, Anfore e commerci nell'alto Adriatico tra VIII e IX secolo, in MEFRM, 120-2, 2008, p. 307-326 ; E. CIRELLI \& E. LO MELE, La cultura materiale di San Severo alla luce delle nuove scoperte archeologiche, in P. RACAGNI (éd.), La basilica ritrovata. I restauri dei mosaici antichi di San Severo a Classe, Ravenna, 2010, p. 39-53, en part. p. 49.

${ }^{17}$ D. CALAON, M. FERRI \& C. BAGATO, SS. Ilario e Benedetto..., op. cit. (n. 64), p. 503 ; E. CIRELLI \& E. LO MELE, La cultura materiale..., op. cit. (n. 116), p. 39-53, en part. p. 49 ; H. PATTERSON, The Soapstone, in J. MITCHELL \& I. L. HANSEN, avec C.M. COUTTS (éd.), San Vincenzo al Volturno 3. The finds from the 1960-1986 excavations, Spoleto, 2001, p. 327-328, fig. 11, n. 1-6.

${ }^{118}$ D. CALAON, M. FERRI \& C. BAGATO, SS. Ilario e Benedetto..., op. cit. (n. 64), p. 503.
} 
observe que dans la phase cistercienne (milieu XIVe-début $\mathrm{XV}^{\mathrm{e}}$ siècle), la majorité des fragments fauniques est issue d'ovins-caprins, puis viennent les bovins, les porcins, la faune sauvage et les animaux domestiques. Bien qu'il s'agisse de données postérieures à la période examinée ici, il semble que l'apport protéique principal au régime des moines se faisait en viandes caprines-ovines et bovines, alors que le poulet et le porc ne devaient être consommés qu'à titre exceptionnel ${ }^{119}$.

En l'absence d'étude des données fauniques de S. Caprasio à Aulla, il est impossible de vérifier quels étaient les choix et les options alimentaires de la communauté monastique alto-médiévale. En supposant que cette grille de lecture sera adéquate, nous proposons de saisir quelles pouvaient y être les valeurs économiques ajoutées aux VII $-\mathrm{XI}^{\mathrm{e}}$ siècle, en partant des huit monnaies découvertes (926-1056), des frappes de Venise (2), Pavie (3), Milan (1), Lucques (1) et Lyon (1). Stratigraphiquement, ces monnaies sont des marqueurs signifiants mais elles ont également des implications d'une autre nature ${ }^{120}$.

$\mathrm{Au}$ haut Moyen Âge, une fois arrivée à l'un des points d'accostage situés entre l'embouchure du fleuve Magra et le golfe de la Spezia-Luni - qui n'était plus le seul choix comme quelques siècles auparavant - toute personne remontant la côte tyrrhénienne par mer ou par terre devait abandonner le secteur côtier pour poursuivre son voyage vers le Nord de la péninsule italienne. Il lui fallait franchir la chaîne des Apennins pour redescendre dans la Plaine du Pô. Les cols septentrionaux se trouvaient à environ $60 \mathrm{~km}$ de Luni, sur la route rectiligne du Magra, dont la pente n'entravait pas le transit des hommes et des animaux, à l'exception d'un court tronçon en amont du confluent de la Vara. Hommes et mules cheminant à une moyenne de $4 \mathrm{~km} / \mathrm{h}$, le meilleur parcours routier quittait le fond de vallée à Sarzana pour monter tout droit, passer la ligne de partage des eaux des Apennins et redescendre vers Aulla, où s'amorçait la ligne droite de fond de vallée du Magra jusqu'à Pontremoli ; de là partaient des embranchements pour Parme et Plaisance. À Aulla confluaient en outre au moins trois autres routes de longue distance : celle qui provenait de Brugnato ; celles qui à travers la vallée de l'Aulella et le col de Tea poussaient jusqu’à Garfagnana, Lucques et Pistoia ; enfin les voies qui à travers les cols de Ospedalaccio et Lagastrello menaient à Reggio d'Émilie et Modène ${ }^{121}$ (fig. 11).

Cela a toujours été le cadre naturel d'Aulla, sur un nœud de communications fondamental entre l'Italie du centre nord et du centre sud. En conséquence, le choix d'Adalbert $I^{\text {er }}$ de fonder dans ce contexte un hôpital, une église et son abbaye en mesure d'accueillir voyageurs et pèlerins, paraît très réfléchi. Constater que les monnaies du haut Moyen Âge ont été émises par des ateliers distincts situés en Italie du Nord et en France ne doit pas surprendre. Il en est de même pour les résultats des examens d’anthropologie physique menés sur les sépultures du cimetière alto-médiéval à l'extérieur du complexe monastique. On n'y a pas enterré de simples paysans comme on l'attendrait, mais des individus issus de souches ethniques variées. Ces tombes pourraient avoir contenu des pèlerins, des voyageurs, des agents de commerce, des marchands et des artisans, les uns attirés par la présence des reliques - dont la mémoire est encore présente dans la tradition populaire locale -, les autres venus mettreà profit leurs compétences ou employer leurs capacités économiques dans de nouvelles zones de travail, sans imaginer que le destin avait prévu pour eux une fin inattendue ${ }^{122}$. La possibilité que les centres monastiques du haut Moyen Âge aient été des lieux et des domaines de convergences aussi diversifiées (prière, production, consommation, gestion et pouvoir) permettra d'ouvrir de nouveaux chapitres de recherche approfondie.

\footnotetext{
${ }^{119}$ F. BERTOLDI \& R. GIACOMELLO, Analisi paleobiologica..., op.cit. (n. 71), p. 272-273.

${ }^{120}$ E. ARSLAN et alii, Indagini..., op. cit. (n. 36), p. 199-205.

${ }^{121}$ Ibid., p. 219-220.

${ }^{122}$ Ibid., p. 220-221.
} 